This item was submitted to Loughborough's Research Repository by the author.

Items in Figshare are protected by copyright, with all rights reserved, unless otherwise indicated.

\title{
Discrete dislocation dynamics modelling of mechanical deformation of nickel- based single crystal superalloys
}

PLEASE CITE THE PUBLISHED VERSION

http://dx.doi.org/10.1016/j.ijplas.2011.07.003

PUBLISHER

(C) Elsevier

VERSION

AM (Accepted Manuscript)

LICENCE

CC BY-NC-ND 4.0

\section{REPOSITORY RECORD}

Huang, Minsheng, Liguo Zhao, and Jie Tong. 2019. "Discrete Dislocation Dynamics Modelling of Mechanical Deformation of Nickel-based Single Crystal Superalloys". figshare. https://hdl.handle.net/2134/10907. 
This item was submitted to Loughborough's Institutional Repository (https://dspace.lboro.ac.uk/) by the author and is made available under the following Creative Commons Licence conditions.

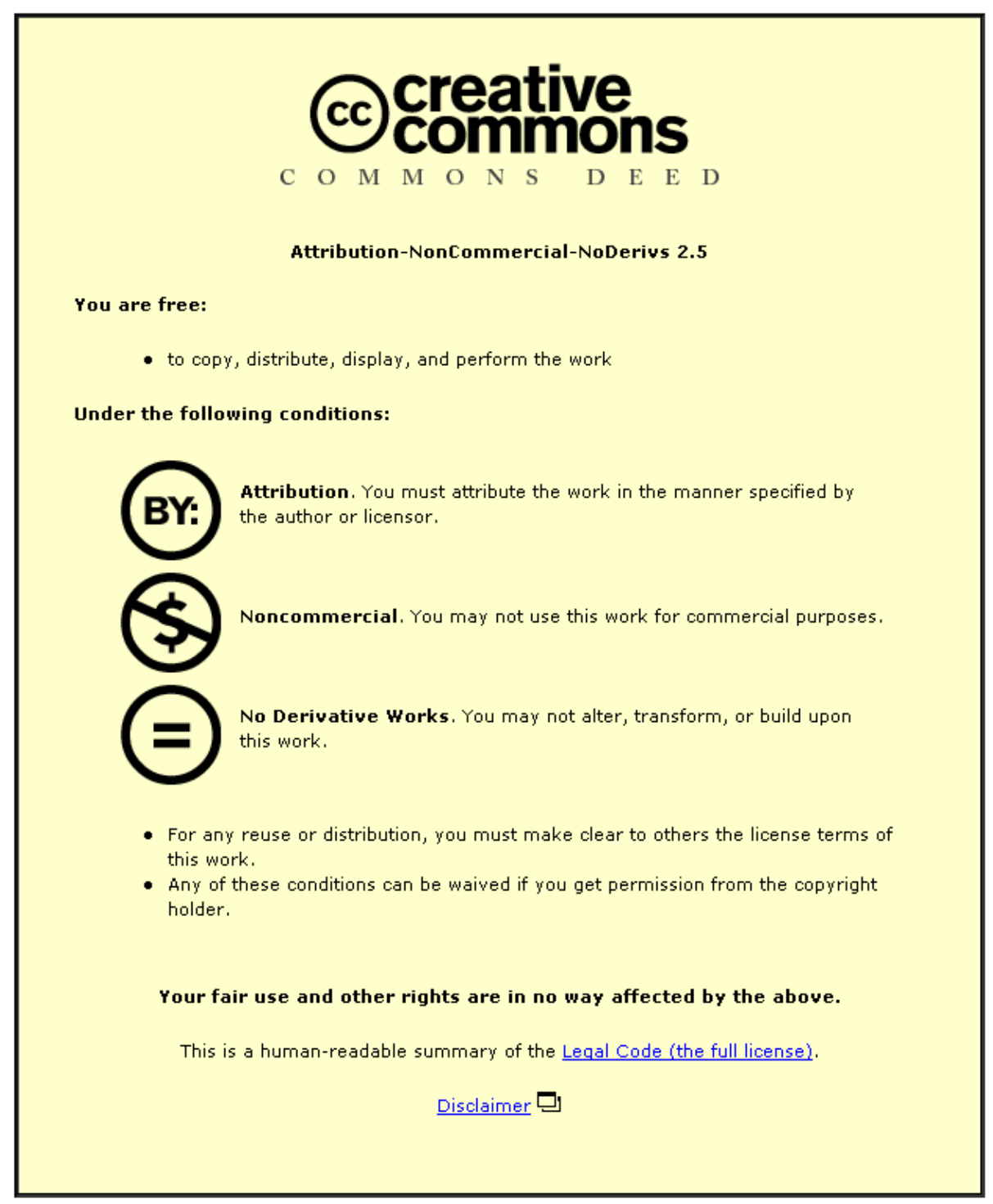

For the full text of this licence, please go to: http://creativecommons.org/licenses/by-nc-nd/2.5/ 


\title{
Discrete Dislocation Dynamics Modelling of Mechanical Deformation of \\ Nickel-Based Single Crystal Superalloys
}

\author{
Minsheng Huang, Liguo Zhao, Jie Tong \\ Mechanical Behaviour of Materials Laboratory \\ Department of Mechanical and Design Engineering, Anglesea Building, Anglesea Road, \\ University of Portsmouth, Portsmouth, PO1 3DJ,UK
}

\begin{abstract}
Discrete dislocation dynamics (DDD) has been used to model the deformation of nickelbased single crystal superalloys with a high volume fraction of precipitates at high temperature. A representative volume cell (RVC), comprising of both the precipitate and the matrix phase, was employed in the simulation where a periodic boundary condition was applied. The dislocation Frank-Read sources were randomly assigned with an initial density on the 12 octahedral slip systems in the matrix channel. Precipitate shearing by superdislocations was modelled using a back force model, and the coherency stress was considered by applying an initial internal stress field. Strain-controlled loading was applied to the RVC in the [001] direction. In addition to dislocation structure and density evolution, global stress-strain responses were also modelled considering the influence of precipitate shearing, precipitate morphology, internal microstructure scale (channel width and precipitate size) and coherency stress. A three-stage stress-strain response observed in the experiments was modelled when precipitate shearing by superdislocations was considered. The polarized dislocation structure deposited on the precipitate/matrix interface was found to be the dominant strain hardening mechanism. Internal microstructure size, precipitate shape and arrangement can significantly affect the deformation of the single crystal
\end{abstract}


superalloy by changing the constraint effect and dislocation mobility. The coherency stress field has a negligible influence on the stress-strain response, at least for cuboidal precipitates considered in the simulation. Preliminary work was also carried out to simulate the cyclic deformation in a single crystal Ni-based superalloy using the DDD model, although no cyclic hardening or softening was captured due to the lack of precipitate shearing and dislocation cross slip in this alloy.

Keywords: Discrete dislocation dynamics; Precipitate shearing; Size effect; Precipitate morphology; Cyclic response.

\section{Introduction}

Single crystal nickel-based superalloys are a class of high temperature and highly corrosion resistant materials, which are widely used in aerospace, marine and power generation industries. They mainly consist of two phases: Ductile face centred cubic (FCC) solid solution (the $\gamma$ phase) and coherent $\mathrm{L}_{2}$-ordered $\mathrm{Ni}_{3} \mathrm{Al}$ precipitates (the $\gamma^{\prime}$ phase). The $\mathrm{Ni}_{3} \mathrm{Al}$ phase is regularly distributed as roughly cuboidal or spherical particles in the matrix. As an important class of structural materials, nickel-based superalloys are usually exposed to high static or cyclic loads in non-ambient environments, and hence a fundamental understanding of their constitutive behaviour is essential for safe life and damage tolerance assessment for fracture critical applications. In the last two decades, considerable work has been carried out to model the constitutive behaviour of the materials, which may be generally classified into the following three categories. The first category treats the material as a homogeneous single phase material where channel width, precipitate size and other specific microstructure features are not explicitly accounted for (McHugh and Mohrmann, 1997; 
Shenoy et al., 2005). The second considers the two-phase nature of superalloys directly (Ohashi et al., 1997; Busso et al., 2000), where the shape, dimensions and properties of both phases are taken into account as model parameters. The third case is a homogenization model statistically introduces the microstructure information at macroscopic level (Fedelich, 1999; 2002; Tinga et al., 2010). All these models are formulated within a continuum framework. Although some interactions between the dislocations and the microstructure may be considered by the strain gradient (Busso et al., 2000) or statistic laws (Fedelich, 1999; 2002), these methods are limited in the study of the interaction between dislocations and the superalloy microstructure (Yashiro et al., 2006), as the statistic laws may not be appropriate at the microscale or nanoscale of precipitates and channels.

Plastic deformation in crystalline metals is a consequence of nucleation, multiplication and motion of a large number of dislocations. Discrete Dislocation Dynamics (DDD) method (Kubin et al., 1992, 1999; Van der Gienssen et al., 1995; Ghoniem et al., 2000; Devincre et al., 2001; Zbib et al., 2002; Arsenlis, et al., 2007; Liu et al., 2009) has been developed to calculate the plastic deformation directly from the evolution of a large number of dislocation segments. The three dimensional (3D) DDD method was originally developed by Kubin et al. $(1992 ; 1999)$ based on the discretisation of dislocations of arbitrary shape into a succession of edge and screw piecewise segments. While in Zbib et al. (1998), the dislocations were treated as a piecewise continuous array of straight line segments with mixed character. On the other hand, Ghoniem et al. $(2000 ; 2002)$ developed a parametric dislocation dynamics (PDD) model by representing dislocations as spatial spline curves connected through dislocation nodes. To improve the computational efficiency in the study of large-scale plastic deformation, Wang et al. (2006) presented a parallel algorithm for the 
3D PDD. Recently, DDD becomes a popular method to study the intrinsic mechanism of mechanical responses of materials (Madec, 2003; 2008). Since an intrinsic length scale, i.e. the Burgers Vector, is included in such a method, it is possible to capture the size effect of internal micro-structure parameters (Vattre et al., 2010). By introducing atomistic information, DDD can predict the experimentally observed plastic anisotropy and tensioncompression asymmetry (Wang et al., 2009; 2010). Also DDD models the dislocation slip, multiplication, annihilation, jog, junction, cross slip (Rhee et al, 1998; Madec et al., 2002; Wang et al., 2007) and climb (Gao et al., 2010) directly, thus it can explicitly capture the interactions between dislocations on different slip systems (Queyreau et al., 2009), the interactions between the dislocations and internal microstructures as well as the formation of different heterogeneous dislocation microstructure such as slip band (Kubin et al., 1992, 1999; Wang et al., 2008; Akarapu et al., 2010) under various loads. Since both the dislocation-internal microstructure interaction and the formation of dislocation microstructures are the keys to understanding the deformation mechanisms, DDD has become an important method to investigate the mechanical behaviour of materials and its intrinsic dislocation mechanisms for both monotonic (Motz et al., 2008) and cyclic loadings (Depres et al., 2004; 2006). For monotonic loading, Groh et al. (2005) performed a three dimensional (3D) DDD simulation of the stress-strain response of particle-reinforced metal matrix composites. They found that the constraint effect on dislocation mobility by the matrix channel width significantly influences its mechanical response. For cyclic loading, Shin et al. (2007) modelled the fatigue behaviour of shearable particle hardened materials by the DDD method, and their results showed that the particle size influences the manner of interaction between dislocations and particles, further the cyclic behaviour. Single crystal nickel superalloys are two-phase materials with a high fraction of phase boundaries, which 
is similar to the particle hardened materials. Thus it is feasible to use the DDD method to simulate the dislocation- $\gamma^{\prime}$ precipitate interaction, the formation of dislocation microstructures and the montonic/cyclic responses of single crystal Ni-based superalloys, which may lead to a more fundamental microscopic understanding of their deformation mechanisms.

The DDD method has been employed to investigate the mechanical behaviour of nickelbased superalloys and their dislocation mechanisms. Rao et al. (2004) calculated the room temperature yield stress of a nickel-based superalloy containing up to $40 \% \gamma^{\prime}$ precipitate by the DDD method. The influence of selected parameters, such as the precipitate size, shape, volume fraction and anti-phase boundary (APB) energy, on the mechanical behaviour of the material, has been investigated. However, the work considered only one slip plane and modelled just one pair of $1 / 2<110>$ dislocations with either screw or $60^{0}$ initial character. Consequently, the effects of strain hardening on the yield stress as a result of dislocation accumulation and interaction between different slip planes and slip systems, could not be adequately captured; and the yield stress was simply assumed as the minimum stress required for the dislocation pair to sweep through the considered glide plane. Within the DDD framework, Yashiro et al. (2006) proposed a back force model to simulate the cutting of dislocation pairs into the precipitate phase and investigated the blocking effect of interfacial dislocation network on precipitate shearing by these dislocation pairs. Vattre et al. (2009) extended Yashiro et al.'s (2006) model by introducing the Kear-Wilsdorf (KW) unlocking stress in the precipitates to account for the influence of high temperature. They predicted the critical resolved shear stress (CRSS) for precipitate shearing at temperature $850^{\circ} \mathrm{C}$ for a range of high precipitate volume fractions ( $40 \% \sim 70 \%$ ), but again considered just 
one dislocation pair on a specific slip plane. On the other hand, Vattre et al. (2010) used the discrete dislocation-continuum model (DCM) to simulate the anisotropic mechanical response of a single-crystal nickel-based superalloy at $1123 \mathrm{~K}$. The uniaxial anisotropic yielding behaviour along [001] and [111] directions was found to be related to the difference in dislocation localization, interfacial dislocation microstructures and dislocation density evolution. However, in this work, the precipitates were assumed to be elastic and no precipitate shearing was taken into account. So far, for nickel based superalloys, the interaction between the internal microstructure features, such as the channel width, precipitate size and morphology, and the dislocation behaviour has not been well understood, hence more systematic studies are required to obtain a thorough understanding of the strengthening mechanisms for this type of alloys. To our best knowledge, no DDD studies have been published in the simulation of cyclic behaviour of the materials.

The objective of this work is to carry out a 3D DDD simulation of mechanical deformation of typically experienced in single crystal Ni-based superalloys, focusing on the interaction between dislocation and internal microstructure. A representative volume cell (RVC) was analysed under strain-controlled loading in the [001] direction, where dislocation sources were distributed on all 12 octahedral slip systems in the matrix channel. Precipitate shearing by superdislocatons was particularly considered in the DDD simulation. Dislocation structure and density evolution were monitored during the simulation. The effects of precipitate shearing, microstructural features and coherency stress on the global stress-strain responses were investigated. Preliminary DDD simulation was also carried out to model the cyclic deformation of the material. 


\section{DDD Simulation Methodology}

\subsection{Simulation model}

As shown in Fig.1, a representative volume cell (RVC), containing a $\gamma^{\prime}$ cubic precipitate of edge length $l$ and $\gamma$ channels of width $d$, was built to represent nickel-based single crystal superalloys such as CMSX4 (Tinga et al., 2010). Both the $\gamma$ and $\gamma^{\prime}$ phases were assumed to be isotropic with the same shear modulus $\mu=37 G P a$ and Poisson's ratio $v=0.37$. The magnitude of the Burgers vector $b$ in both phases was chosen to be $0.25 \mathrm{~nm}$. An uniaxial load was applied to the RVC in the [001] direction (z axis) under strain-controlled condition. Periodic boundary condition (PBC) was applied to the RVC to satisfy the mechanical equilibrium, dislocation flux balance and dislocation line continuity conditions at the boundaries. As the PBC condition is applied to the RVC, artificial self-annihilation of dislocation loops occurs (Madec et al., 2003). To avoid this, the RVC needs to have a nonperfect cubic shape, which also leads to a non-perfect cubic shape for the precipitate if the channel width is kept fixed. For instance, at a channel with $d=250 \mathrm{~b}$ and a precipitate volume fraction $f=63 \%$, the dimensions of the RVC and the precipitates are $1670 b \times$ $1750 b \times 1830 b$ and $1420 b \times 1500 b \times 1580 b$, respectively. The size of precipitate given in this paper is therefore an average value of the three sides.

\subsection{Peach-Kohler force calculation}

Following the DDD framework developed by Zbib et al. (1998; 2002), all dislocation lines and loops of arbitrary shape were discretized into short line segments and the evolution of each dislocation segment is determined by the motion of dislocation nodes. The Peach-Kohler 
(PK) force $F_{i}$, which controls the motion of a given dislocation segment $i$, may be expressed as:

$$
F_{i}=\left(\sum_{j=1}^{N-1} \sigma_{\mathrm{j}}+\sigma^{a p p}\right) b_{i} \times \xi_{i}
$$

where $\sum_{j=1}^{N-1} \sigma_{\mathrm{j}}$ is the long-range stress caused by all the other $(\mathrm{N}-1)$ dislocation segments, $\sigma^{a p p}$ is the externally applied stress, $b_{i}$ and $\xi_{i}$ are the Burgers vector and line sense vector of segment $i$, respectively.

For each dislocation segment, the free glide velocity of dislocation $v_{i}$ during a simulation time step may be determined as:

$$
v_{i}=\left\{\begin{array}{ccc}
0 & \text { if } & \operatorname{abs}\left(F_{i}^{\text {glide }}\right) \leq a b s\left(\tau_{F} b_{i}\right) \\
\frac{F_{i}^{\text {glide }}+\tau_{\text {Int }} b-\tau_{F} \operatorname{sign}\left(F_{i}^{\text {glide }}\right) b_{i}}{B} & \text { if } & \operatorname{abs}\left(F_{i}^{\text {glide }}\right)>\operatorname{abs}\left(\tau_{F} b_{i}\right)
\end{array}\right.
$$

where $F_{i}^{\text {glide }}$ is the glide component of the Peach-Kohler force $F_{i}$ on the slip plane, $\tau_{F}$ a constant friction stress, $\tau_{\text {int }}$ an initial internal stress introduced by lattice mismatch between $\gamma / \gamma^{\prime}$ phases (i.e., the coherency stress field) and anti phase boundary (APB) and $B$ the viscous drag coefficient.

For efficient computation of the PK force $F_{i}$ (Eq.(1)), the RVC was further divided into a series of subcells (Zbib et al., 1998). For dislocations within the same and immediate neighbouring subcells, their contribution to PK force on dislocation $i$ was computed at the centre of the dislocation segment $i$ directly. While, for dislocations in other remote subcells, their contributions to PK force were computed only at the centre of the subcell containing the dislocation segment $i$. The analytical de Wit formulation (de Wit, 1967) was used to calculate the interaction force. In addition, the dislocation interaction stress between periodic RVCs was calculated using the "multipolar expansion" method (Zbib et al., 1998), 
which reduces the computational cost from the order of $N^{2}$ to $N \log (N)$. Also, a parallel long-range stress calculation was realized by the OpenMP interface to further improve the computational efficiency.

When a dislocation glides in the $\gamma$ channel, the solute atoms have a hindering effect on it. This is a chemical solid-solution force, which was taken into account in the DDD simulation by a friction stress $\tau_{F}=200 M P a$ opposing the dislocation motion in Eq.(2). This chemical force is considered as the elementary mechanism controlling the critical resolved shear stress (CRSS) of the $\gamma$ phase, which is in the order of hundreds MPa (Vattre et al., 2009). In Rao et al.'s (2004) DDD simulation on single crystal superalloy, the friction stress was assigned in the range of $100-500 \mathrm{MPa}$. In the present simulation, the chosen $200 \mathrm{MPa}$ is in the same order as Vattre et al. (2009) and Rao et al. (2004), which also gives the initial yielding stress of 900MPa coincident with that of CMSX-4 (Tinga et al., 2010). The dislocation-precipitate interactions included Orowan looping and precipitate shearing (Fedelich, 2002), while dislocation climb was neglected in the DDD simulations. The Orowan looping is a direct result of dislocation motion in the channel, thus no special treatment is needed. For precipitate shearing, it can occur either by dislocation pairs or by Superlattice Intrinsic Stacking Faults (SISF) (Fedelich, 1999; Tinga et al., 2010). Since partial dislocations and dislocation dissociation were not introduced in the DDD program, the cutting of coherent intermetallic $\gamma^{\prime}$ precipitates was simulated by the glide of $\langle 110\rangle$ superdislocations. Such superdislocations are pairs of $\frac{1}{2}\langle 110\rangle$ dislocations, namely a leading dislocation and a trailing dislocation, separated by an antiphase boundary (APB) (Veyssiere and Saada, 1997; Yashiro et al., 2006; Vattre et al., 2009). When the leading dislocation cuts into the $\gamma^{\prime}$ phase, the $\mathrm{L}_{2}$ order of atoms in the glide plane is destroyed, thus an APB with energy density $\chi_{A P B}$ 
is produced in the swept area behind the leading dislocation. As a result, a strong back stress $\tau_{A P B}=-\chi_{A P B} / b$ corresponding to the internal microstructure stress $\tau_{\text {Int }}$ in Eq. (2) exerts on the leading dislocation. When the trailing dislocation shears into the precipitate following the leading one, the $\mathrm{L}_{2}$ order of atoms is recovered and the APB only exists in the glide plane between the leading and trailing dislocations. Thus a stress on the trailing dislocation $\tau_{A P B}=\chi_{A P B} / b$ pointing towards the leading dislocation is introduced. It should be mentioned that this stress only exerts on trailing dislocation when it enters into the precipitate. By experimental observation, Sun et al. (1999) suggested that $\chi_{A P B}$ in the $\{111\}$ octahedral slip plane is $144 \pm 20 \mathrm{~mJ} / \mathrm{m}^{2}$. Consequently, in the present paper, $\chi_{A P B}$, was taken as $162.5 \mathrm{~mJ} / \mathrm{m}^{2}$.

The screw dislocations in the $\gamma^{\prime}$ precipitate on the octahedral slip planes can cross slip to the $\{010\}$ cubic planes and introduce the Kear-Wilsdorf (KW) locking (Kear and wilsdorf, 1962). These KW locks then act as obstacle points to the dislocation motion and is believed to be the mechanism for the anomalous flow stress dependence on temperature. Devincre et al. $(1997 ; 1999)$ simulated the formation and the destruction of KW locks in $\gamma^{\prime}$ precipitate by DDD method. In the present paper, the KW locks were not simulated directly but considered by a $\mathrm{KW}$ unlocking stress $\tau_{K W}$, which acts as a friction stress in the precipitate corresponding to the $\tau_{F}$ in Eq. (2), and may be expressed as (Vattre et al., 2009; Demura et al., 2007):

$$
\tau_{K W}=\sqrt{\mu B f_{D} \frac{b}{l_{s}}} \exp \left(-\frac{\Delta H_{0}}{2 k T}\right)
$$

where $k$ is the Boltzmann constant, $f_{D}$ a Debye frequency factor, $l_{s}$ the screw segment length, $T$ the absolute temperature and $\Delta H_{0}$ the activation enthalpy for $\mathrm{KW}$ locks. 
To simulate precipitate shearing by a series of superdislocations, it is necessary determine whether the dislocation segment entering into the $\gamma^{\prime}$ phase is a leading dislocation or a trailing dislocation. The method used in the DDD simulations is described as follows. For a dislocation segment $i$ in the precipitate, two glide forces are defined as:

$$
F_{a p p}=\left(\sigma_{a p p} \cdot b_{i} \times \xi_{i}\right) \cdot\left(n \times \xi_{i}\right) \text { and } F_{\text {Ina }}=\left(\sigma_{\text {Ina }} \cdot b_{i} \times \xi_{i}\right) \cdot\left(n \times \xi_{i}\right)
$$

where $n$ is the normal vector of the slip plane of dislocation segment $i, F_{a p p}$ is the glide force induced by the external loads $\sigma_{a p p}$ and $\sigma_{\text {Ina }}$ is the stress at the centre of dislocation segment $i$ introduced by an interacting dislocation. As shown in Fig.2, the interaction dislocation for segment $i$ is defined as the closest neighbouring dislocation segment $j$. It lies in the same slip plane and has the same character as the segment $i$ (the same Burgers vector and line direction), but belongs to a different dislocation loop. If the interacting dislocation cannot be found, the stress $\sigma_{\text {ina }}=0$. Otherwise, if $F_{a p p} . F_{\text {Ina }}<0$ and $\operatorname{abs}\left(F_{\text {Ina }}\right)>\alpha \chi_{A P B}$, the dislocation segment $i$ is a trailing dislocation while it will be a leading dislocation if $F_{a p p} . F_{I n a} \geq 0$ and $a b s\left(F_{I n a}\right)>\alpha \chi_{A P B}$. Here, considering the balance of the interaction force between leading and trailing dislocations and the configuration force induced by the APB, the total force on a trailing (leading) dislocation introduced by the whole leading (trailing) dislocation will be equal to $\chi_{A P B}$. As the interaction dislocation defined above is only one segment of the leading (trailing) dislocation, the interaction force $F_{\text {Ina }}$ will be less than the total force $\chi_{A P B}$, and consequently $\alpha$ should be less than 1 . However, $\alpha$ cannot be too small, otherwise the third dislocation of pile-ups near the $\gamma / \gamma^{\prime}$ interface, which is further away, may be misjudged as a trailing dislocation although it is not. As verified by a series of calculations, this misjudgment can be eliminated by choosing $\alpha=$ 0.25 which has been used throughout our DDD simulations. 
Due to the lattice mismatch between the $\gamma$ and $\gamma^{\prime}$ phases, an internal coherency stress field is normally presented in Ni-based superalloys. The parameter $\delta=2\left(a_{\gamma^{\prime}}-a_{\gamma}\right) /\left(a_{\gamma^{\prime}}+a_{\gamma}\right)$ is generally used to quantify the lattice mismatch, where $\mathrm{a}_{\gamma^{\prime}}$ and $\mathrm{a}_{\gamma}$ are the lattice parameters for the precipitate and the matrix channel, respectively. According to Brien et al. (2001), the misfit stresses in the channels can be written as

$$
\begin{cases}\sigma_{22}^{m}=\sigma_{33}^{m}=K \delta & \text { in channel normal to } x_{1} \text { axis } \\ \sigma_{11}^{m}=\sigma_{33}^{m}=K \delta & \text { in channel normal to } x_{2} \text { axis } \\ \sigma_{11}^{m}=\sigma_{22}^{m}=K \delta & \text { in channel normal to } x_{3} \text { axis }\end{cases}
$$

where $K$ is the bulk modulus. The misfit stress in the precipitates can be obtained from stress equilibrium, i.e., zero resultant stress in the RVC. At temperature $850^{\circ} \mathrm{C}$, the lattice mismatch $\delta$ is negative, which causes a biaxial compressive stress state in the matrix channels according to Eq.(5). While the precipitate is subjected to tensile misfit stress. Here, the lattice misfit parameter is chosen as $\delta=-1.1 \times 10^{-3}$ at $850^{\circ} C$ (Tinga et al., 2010). In the DDD simulation, the coherency stress field (5) is included by assigning an initial internal stress field to the RVE and is considered as an internal stress $\tau_{\text {int }}$ in Eq.(2).

\subsection{DDD short range interaction and time step scheme}

Initial dislocation sources were assigned in the $\gamma$ matrix only. Under mechanical loading, the dislocations not only glide under the PK force but also interact with each other by short range interactions. These short-range interactions, such as dislocation annihilation, jogs and junctions, were monitored at every time step by a series of constitutive laws (Rhee et al., 1998). The screw dislocation cross slip was determined numerically using a Monte-Carlo type simulation (Zbib et al., 2002, Shin et al, 2007). To simulate the dislocation multiplication, initial dislocation Frank-Read sources with a given density was randomly distributed on the 12 octahedral slip systems in the FCC matrix channel. As no dislocation 
motion was found on cubic slip planes by the TEM observations (Wang et al., 2009), cubic slip systems were not considered. All the initial dislocation sources have a length twice the channel width $d$, so the dislocation sources can be active even before reaching the channel width-controlled Orowan threshold stress $\tau^{\mathrm{Or}}=\mathrm{k} \mu \mathrm{b} / \mathrm{d}$ ( $k$ a pre-factor accounting for the dislocation line tension). If the length of initial dislocation sources is smaller than the channel width $d$, the influence of $d$ on the initial yielding stress $\sigma_{i n t}^{y}$ will be shielded and the $\sigma_{\text {int }}^{y}$ can be significantly and unrealistically elevated.

The equations in the DDD system are solved using a forward explicit integration algorithm. At the $n t h$ loading step, i.e., from time $t_{n}$ to time $t_{n+1}$, the dislocation glides under a constant applied stress $\sigma_{n}^{a p p}$, which produces a plastic strain increment $\Delta \epsilon_{P}$. At the next loading step, i.e., from time $t_{n+1}$ to time $t_{n+2}$, the applied stress is updated as $\sigma_{n}^{a p p}+$ $E \dot{\varepsilon}\left(t_{n+1}-t_{n}\right)-E \Delta \epsilon_{P}$, where $E$ is the Young's modulus and $\dot{\varepsilon}$ is the strain rate. Unless otherwise specified, the strain rate was chosen to be $1 / \mathrm{s}$ considering the computational cost. To choose the time increment, a truncated dislocation velocity $V_{\text {trun }}=0.1 V_{\text {shear }}$ was used, where $V_{\text {shear }}$ is the shear wave speed. If the ratio $\frac{\Delta \epsilon_{P}}{\dot{\varepsilon}\left(t_{n+1}-t_{n}\right)}=\frac{\Delta \epsilon_{P}}{\dot{\varepsilon} \Delta t}$ is less than 0.01 , it is believed that most dislocations cannot glide efficiently under the applied stress $\sigma_{n}^{a p p}$, and thus the next time increment will be doubled $2 \Delta t=2\left(t_{n+1}-t_{n}\right)$ to speed up the computation. This type of time increment scheme can deal with the problem of high dislocation speed oscillation near the $\gamma / \gamma^{\prime}$ interfaces more efficiently and does not influence the simulation accuracy.

\section{Results and Discussion}


DDD simulations were carried out at a temperature of $850^{\circ} \mathrm{C}$ to reflect the working environment of nickel superalloys. Also at this temperature, no rafting will occur for the applied strain level. The RVE was loaded, at a strain rate of $1 / \mathrm{s}$, up to $2.0 \%$ strain which allows the precipitate shearing to come into effect. The RVC was divided into $12 \times 12 \times 12$ subcells and 4-order multipolar expansion of dislocations (Zbib et al., 1998) was adopted in the simulation.

\subsection{Monotonic stress-strain response and its mechanism}

For a RVE with a precipitate size of $1420 b \times 1500 b \times 1580 b$ and a channel width of $250 \mathrm{~b}$, the simulated stress-strain response in [001] direction is shown in Fig.3 for the cases with and without considering precipitate shearing. The volume fraction of $\gamma^{\prime}$ phase in the RVE is calculated to be $f=63 \%$. It can be seen from Fig. 3 that, without considering precipitate shearing, only two-stage deformation can be observed: the initial elastic stage $A$ and the hardening stage $B$. During stage A, since the initial dislocation sources in the channels are inactive or just slip slightly towards the $\gamma / \gamma^{\prime}$ interfaces, both the matrix and precipitate phases remain elastic. When the resolved shear stress on the slip systems becomes larger than the Orowan stress $\tau^{\mathrm{Or}}=\mathrm{k} \mu \mathrm{b} / \mathrm{d}$, where $d$ is the channel width and $k$ a pre-factor accounting for the dislocation line tension, the initial yielding occurs and hardening stage $B$ begins. At the beginning of the hardening stage, no dislocation pile up is observed in the channel. With the increase of applied load, pile-ups are formed around the interface, which decreases the effective width of the channel and increases the hardening rate. However, when precipitate shearing is considered, a softening stage or a steady stage $C$, following the elastic stage $A$ and the hardening stage $B$, is observed in the stress-strain response, which is consistent with the experimental observation (Tinga et al., 2010; Busso et al., 2000). This 
seems to suggest that the precipitate shearing by dislocations, which introduces plastic deformation in the precipitates, is the mechanism for the strain softening in the third stage. Also, it is recommended that the precipitates should be treated as inelastic, instead of elastic in continuum FE studies, in order to accurately simulate the plasticity-associated softening behaviour of the material.

Fig.4 shows the dislocation microstructure at $0.2 \%$ plastic strain, which is within the second hardening stage. In the simulation, eight out of the twelve octahedral slip systems are active,

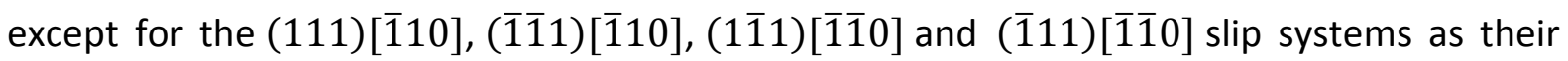
Burgers vectors are normal to the resolved shear stresses on the slip planes. Both dislocation junctions and jogs can be observed in the channels and they contribute to the strain hardening. Most dislocation segments are deposited on the $\gamma / \gamma^{\prime}$ interfaces, with either a screw character or a mixed character (Burgers vector at $\pm 60^{\circ}$ to the dislocation line direction). Similar to the TEM observation of Tian et al. (2000), these deposited dislocations form a kind of network of dislocation lines which are normal to each other. As indicated by the black arrow in Fig.4, the deposited dislocations have a strong blocking effect on the subsequent motion of bypassing dislocations, which contributes to strain hardening. In particular, ' $V$ '-shaped dislocations are formed on the interfaces, which is the result of collinear annihilation of dislocations, with the same Burgers vector, on different slip planes (Devincre et al., 2007). For example, if two dislocations glide in the channels on the slip systems (111)[ $\overline{1} 10]$ and $(\overline{1} \overline{1} 1)[\overline{1} 10]$, respectively, they can annihilate each other at the intersection line of these two slip systems, which generates two 'V'-shaped immobile dislocation debris on the precipitate interfaces as shown in Fig.4. Since the mobile parts of 
these two dislocations disappear, it leads to a decrease of dislocation sources and plastic deformation and consequently increases the strain hardening.

The evolution of total dislocation density $\rho^{\text {tot }}$, junction dislocation density $\rho^{\text {jun }}$ and jog dislocation density $\rho^{\text {jog }}$ without considering precipitate shearing are plotted in Fig.5 as a function of plastic strain $\varepsilon_{\mathrm{p}}$. The junction dislocation density is calculated by averaging the total length of junction segments over the RVC volume, while the jog dislocation density is the average of the total length of dislocations with jogs over the RVC volume. It can be seen that both junction and jog dislocation densities make negligible contributions to the total dislocation density within the applied strain level. For example, as shown in Fig.5 for simulations without considering dislocation shearing, the density of dislocations with jogs and junctions at $0.4 \%$ plastic strain are $5.2 \times 10^{11} \mathrm{~m}^{-2}$ and $6 \times 10^{13} \mathrm{~m}^{-2}$, which are two to four orders less than the total dislocation density $1.25 \times 10^{15} \mathrm{~m}^{-2}$. This is also true for the simulations with dislocation shearing. In this case, at $0.4 \%$ plastic strain, the density of dislocation with jogs and junctions are $4.5 \times 10^{11} \mathrm{~m}^{-2}$ and $5.6 \times 10^{13} \mathrm{~m}^{-2}$ while the total dislocation density is $1.0 \times 10^{15} \mathrm{~m}^{-2}$. This suggests that the dislocation junctions and jogs play minor roles in the strain hardening behaviour. In addition, from the dislocation microstructure, it can be seen that only few ' $\mathrm{V}$ '-shaped dislocations are formed, therefore their contribution to strain hardening may be limited. To quantify the dislocation network deposited on the interfaces, an equivalent polarised dislocation density $\alpha^{\text {eq }}$ which may be defined as:

$$
\alpha_{e q}=\operatorname{sign}\left(\alpha_{11}\right) \sqrt{\alpha_{i j} \alpha_{i j}}
$$

where $\alpha_{i j}$ is the Nye tensor. For discrete dislocation simulations, the Nye tensor may be expressed as (Arsenlis and Parks, 1999): 


$$
\alpha_{i j}=\frac{1}{V} \sum_{k=1}^{N} l^{k} b_{i}^{k} t_{j}^{k}
$$

where $N$ is the total number of segments, $\mathrm{V}$ the volume of the RVC, and $l^{k}, b^{k}$ and $t^{k}$ are the length, the unit vector and the Burgers vector of the dislocation segment $k$, respectively. The distribution of the equivalent polarised dislocation density $\alpha^{\mathrm{eq}}$ across the [001] horizontal channel is plotted in Fig.6 at the $1.5 \%$ applied strain level, where the $x$-axis represents the normalised distance $(x / d)$ across the width of the channel. Interestingly, it seems that the equivalent polarised dislocation density increases drastically towards the interfaces (i.e. $x / d=0$ and 1), where the dislocation network forms. These polarized interfacial dislocations can introduce high internal back stresses which reduce the PeachKohler force and subsequently the mobility of dislocations in the channels. This suggests that the dislocation network formed at the interface is the major source for strain hardening, amongst other mechanisms such as the formation of dislocation junctions and jogs and the ' $V^{\prime}$-shaped configuration. In addition, it should be mentioned that, similar to the work in literatures (Vattre et al., 2009; Rao et al., 2004 and Shin et al., 2007), the difference in elastic properties between the two phases were not considered, although it is very small (shear modulus 100.2 GPa for $\gamma$ and 105.2GPa for $\gamma^{\prime}$ (Tinga et al., 2010)). This difference will introduce both an image stress on the dislocation and the inhomogeneous stress field, which are expected to increase the hardening effect of the material. We are currently updating the DDD framework which can consider the difference in elastic properties between the two phases to investigate such effects quantitatively.

In the third stage $C$, precipitate shearing by superdislocations occurs, as evidenced from the dislocation microstructure in Fig.7 at $0.8 \%$ plastic strain level. A series of superdislocations cutting into the precipitate can be easily identified, which consist of leading and trailing 
dislocations. When the superdislocations shear into the precipitate, they can bow out with a larger curvature radius than those in the channels due to the loss of confinement imposed by the narrow channels. For dislocation gliding in the channel, they need to overcome a high internal back stress to bypass the dislocation network already deposited on the interface. However, for superdislocations gliding in precipitates, although they need to cut through the dislocation network on the interface, most part of these superdislocations is far from the deposited dislocation network and experience much smaller internal obstruction. As a result, when a superdislocation forms and cuts into the precipitate, the two dislocations will form a superdislocaton which can relatively easily sweep a large area in precipitates and produce a high plastic strain increment for a given time step. As long as the plastic strain increment is larger than or equal to the applied strain increment, softening occurs. On the other hand, the leading dislocation of a superdislocation is generally the Orowan dislocation loop already deposited on the interface. Therefore, when a superdislocation cuts through the precipitate, the leading Orowan dislocation loop disappears, resulting in a decreased density of dislocations deposited on the interface. This can be seen from the dislocation density evolution in Fig.5, where the dislocation density increases linearly during the hardening stage while decreases or increases only slightly during the softening stage when precipitate shearing comes into effect. This decrease of interfacial deposited dislocations will reduce the internal back stress in the channels, and further weaken the strain hardening of the matrix phase. The cross slip is also believed to be a mechanism to reduce the hardening response. However, in our simulation, the cross slip events are very limited for the considered [001] tensile loading, as the signs of the resolved shear stresses are opposite to each other for all six pairs of cross-slip systems (Tinga et al., 2010). 


\subsection{Effect of the internal microstructure scale}

The stress-strain response of the RVC is shown in Fig.8a for three selected channel widths $d=\{250,300,350\} b$ at a fixed precipitate size of $1420 b \times 1500 b \times 1580 b$, corresponding to a volume fraction of $63 \%, 58 \%$ and $53 \%$, respectively. As seen in Fig. 8 a, at the strain hardening stage, the narrower the channel width, the higher the yielding stress and the hardening rate. In addition, at the precipitate shearing stage, the stable flow stresses are also significantly higher for the material with smaller channel width.

To explain the above phenomenon, the dislocation density evolution is plotted in Fig.8 as a function of the plastic strain $\varepsilon_{p}$ (b) and the total applied strain $\varepsilon(\mathrm{c})$, respectively. From Fig.8(b), it seems that, at the plastic hardening stage, the dislocation density evolution fits very well with the following equation:

$$
\frac{\partial \rho}{\partial \varepsilon_{p}}=\frac{2 \sqrt{6}}{d b}
$$

This means that, at the hardening stage, the ratio of dislocation density to plastic strain is controlled by the channel width $d$. In fact, Eq.(8) can be obtained as follows. When a dislocation with Burgers vector $\frac{b}{\sqrt{2}}[0 \overline{1} 1]$ glides on the slip plane (111), as shown in Fig.9, its length will be increased by $2 l$, a result of the two segments deposited on the $\gamma / \gamma^{\prime}$ interface, at a given time step $\Delta \mathrm{t}$. Consequently, the plastic strain increment during this time step can be express as:

$$
\Delta \varepsilon_{p[001]}=\frac{\Delta A}{V} n_{[001]} b_{[001]}=\frac{l d}{V} \frac{1}{\sqrt{3}} \frac{b}{\sqrt{2}}
$$

where $\Delta A$ is the swept area by dislocation motion during the time step, $V$ is the volume of the RVC, $n_{[001]}$ and $b_{[001]}$ are the components of the unit normal vector of (111) slip plane 
and Burgers vector along the [001] direction, respectively. As a result, the dislocation density increment during this time step $\Delta$ t can be written as:

$$
\Delta \rho=\frac{2 l}{V}
$$

Dividing Equation (10) by Eq. (9) leads to Eq. (8). Since no precipitate size is specified in obtaining Eqs.(8-10), Eq.(8) is valid for materials with any precipitate size. It further proves that, at the hardening stage, most dislocation segments are deposited on the $\gamma / \gamma^{\prime}$ interface and the storage of forest dislocations (i.e. dislocation junction) in the channels is negligible. The tangent modulus of the hardening stage can be calculated by:

$$
E_{t}=\frac{\dot{\varepsilon} \Delta t-\Delta \varepsilon_{p}}{\dot{\varepsilon} \Delta t} E=\frac{\dot{\varepsilon} \Delta t-\mathrm{db} \Delta \rho / 2 \sqrt{6}}{\dot{\varepsilon} \Delta t} E .
$$

As shown in Fig.8(c), the dislocation density increment $\Delta \rho$ for a given time step $\Delta \mathrm{t}$ is basically the same for different channel width, and consequently, according to Eq.(11), the material with a narrower channel width $d$ would have a higher hardening modulus.

For the third softening stage, the stress increment $\Delta \sigma$ during a specified time step $\Delta t$ should be smaller than or equal to 0 , i.e.

$$
\Delta \sigma=\mathrm{E}\left(\dot{\varepsilon} \Delta \mathrm{t}-\Delta \varepsilon_{\mathrm{p}}\right)=\mathrm{E}\left(\dot{\varepsilon} \Delta \mathrm{t}-\Delta \varepsilon_{\mathrm{p}}^{\text {loop }}-\Delta \varepsilon_{\mathrm{p}}^{\text {cut }}\right) \leq 0
$$

where $\Delta \varepsilon_{\mathrm{p}}^{\text {loop }}$ and $\Delta \varepsilon_{\mathrm{p}}^{\text {cut }}$ are the plastic strains caused by dislocation overlooping and cutting the precipitates, respectively. Obviously, to produce the same magnitude of $\Delta \varepsilon_{\mathrm{p}}^{\text {loop }}$, a larger applied stress $\sigma$ is needed for superalloys with smaller channel width according to the Orowan formula. On the other hand, since Eq. (11) is valid for the hardening stage which has dislocation overlooping but no shearing, it can be further written as:

$$
E\left(\dot{\varepsilon} \Delta t-\Delta \varepsilon_{\mathrm{p}}^{\text {loop }}\right)=E_{t} \dot{\varepsilon} \Delta t
$$


Substituting Eq. (13) into Eq. (12), the condition for the third softening stage to occur can be derived as:

$$
\Delta \varepsilon_{\mathrm{p}}^{\mathrm{cut}} \geq E_{t} \dot{\varepsilon} \Delta t
$$

For smaller channel width, as the tangent modulus $E_{t}$ tends to be higher, more dislocation cutting events are required in order to produce a larger $\Delta \varepsilon_{\mathrm{p}}^{\text {cut }}$ to satisfy the above condition (14) for the softening stage. However, in narrower channels, dislocation motion and formation of superdislocation are more difficult and thus prohibit the occurrence of further dislocation cutting, which consequently leads to a higher flow stress in the third stage.

The stress-strain responses of the RVC is shown in Fig.10 for three selected precipitate sizes $l=\{750,1050,1420\} b$ at a constant channel width $d=250 b$, corresponding to a volume fraction of $45 \%, 55 \%$ and $63 \%$, respectively. As shown in Fig.10, both the hardening modulus at the second stage and the flow stress at the third stage increase with the increase of precipitate size. Since the channel width remains the same, so does the mobility of the dislocations in the channels. As a result, a dislocation tends to sweep the same area at a given time step irrespective of the precipitate size. As the RVC volume is larger for larger precipitates, the plastic strain increment induced by the dislocation will be reduced according to Eq.(9), leading to a higher second-stage hardening rate (or a higher tangent modulus $\left.E_{t}\right)$. For a reduced precipitate size, the radius of the dislocation loop deposited on the precipitate surface becomes smaller, which will enhance the self-interaction or contraction of the loop leading to increased line tension. Consequently, the superdislocations consisting of these dislocation loops have a lower critical stress to overcome for shearing into the precipitate. When a dislocation shears through a smaller precipitate, it also produces a larger plastic strain increment $\Delta \varepsilon_{\mathrm{p}}^{\text {cut }}$ according to Eq.(9). 
Therefore, for smaller precipitate size, less precipitate shearing events are required to meet the condition (14) at the softening stage, hence a lower flow stress is expected.

For a fixed precipitate volume fraction (63\%), the stress-stress responses are plotted in Fig.11 for three selected RVC sizes, which correspond to a channel width of $d=$ $\{200,250,350\} b$ and a precipitate size of $l=\{1200,1500,2100\} b$, respectively. It can be seen from Fig.11 that, at the second hardening stage, both the $0.2 \%$ proof yielding stress $\left(\sigma_{y}=\{1100,1050,955\} \mathrm{MPa}\right)$ and the hardening rate increase with the decrease of the RVC size. In fact, the channel width and the precipitate size decrease simultaneously with decrease of the RVC size. The decreased channel width tends to increase the hardening rate and the yielding stress, while the decreased precipitate size has the opposite effect. Thus it seems that decreased channel width contributes, predominantly, to the increase of the yielding stress and the hardening rate. For the third softening stage, the situation is more complex. The flow stress at this stage is at the same level for the case with $d=200 b$, $l=1200 b$ and the case with $d=250 b, l=1500 b$, which might be due to the opposite effects produced by the channel width and the precipitate size. However, the flow stress is clearly smaller for the case with $d=350 b$ and $l=2100 b$, which indicates that the effect of channel width becomes more significant than that of precipitate size with the increase of the RVC size. Using the gradient-dependent crystallographic constitutive formulation, Busso et al. (2000) also investigated the size effect of the stress-strain response of a single crystal superalloy CMSX4 based on the FE analyses. Their results, however, showed a monotonic increase of the flow stress in the third stage with the decrease of the RVC size. This might be due to their assumption that the plastic deformation only occurs in the $\gamma$ channel while the $\gamma^{\prime}$ precipitate remains elastic with no shearing by dislocations. In fact, the dislocation cutting 
into the precipitate can reduce the dislocation accumulation at the $\gamma / \gamma^{\prime}$ interface and decrease the geometrically necessary dislocation density in the channel, leading to a reduced flow stress. Thus, the size effect on mechanical response is not only associated with the deformation gradient in the channel but also related to the dislocation shearing of precipitate which should be considered in simulations.

\subsection{Effect of precipitate morphology}

To investigate the precipitate morphology effect, simulations were carried out for the RVC containing spherical and cuboidal precipitates with a fixed volume fraction of $f=55 \%$. The spherical precipitate has a radius of $r=700 \mathrm{~b}$ while the cuboidal one has a size of $L=1130 b$, resulting the same volume from both cases. The spherical precipitates have a body faced cubic $(\mathrm{BCC})$ arrangement in order to accommodate the high volume fraction. The stress-strain responses for the two cases are similar in the elastic and the softening stage, although in the hardening stage, the hardening rate and the yielding stress are higher larger for spherical precipitate than those for cuboidal one (Fig.12). The yield stress $(0.2 \%$ proof strain) is about $1130 \mathrm{MPa}$ for spherical precipitates and 970MPa for cuboidal ones. According to the Taylor hardening $\tau=\tau_{0}+\alpha \mu b \sqrt{\rho}$, highly accumulated dislocation density $\rho$ can lead to an increased hardening rate. However, the DDD simulated dislocation density for spherical precipitates is much smaller than that for cuboidal ones, which cannot rationalise the higher hardening rate for spherical case using this classical storage-recovery theory. For

a fixed precipitate volume fraction, the narrowest distance $d_{\min }$ between neighbouring precipitates is much smaller for spherical-shaped precipitates than that for cuboidal-shaped ones. According to the Orowan formula $\tau^{\mathrm{Or}}=\mathrm{k} \mu \mathrm{b} / \mathrm{d}_{\min }$, a much higher-level resolved shear stress is required to drive the dislocation to glide through these narrowest parts of 
matrix channels for spherical precipitates. As these narrowest parts of matrix channels are periodically distributed, they tend to produce an effective blockage to the motion of dislocations, as shown in Fig.13. Therefore, the mobility of dislocations is lower for the spherical precipitates, leading to lower plastic deformation and higher hardening rate when compared to that of cuboidal precipitates.

For the RVC model presented in Fig.1, the precipitates are arranged in an ideal simple cubic (SC) manner. Simulations were also carried out for a BCC arrangement and the results are compared in Fig. 14 against that for SC arrangement. For both cases, the precipitate size and the volume fraction were kept the same, i.e., $\mathrm{l}=1500 \mathrm{~b}$ and $\mathrm{f}=63 \%$, respectively, and the initial dislocation source densities are set to be $\rho_{\text {sou }}=1.1 \times 10^{13} \mathrm{~m}^{-2}$. It can be seen that, in the second hardening stage, the strain hardening rate, as well as the $0.2 \%$ proof yield stress, for BCC arrangement is significantly higher than that for SC arrangement. This is because the precipitates in the BCC arrangement tend to block some of the infinite long channels existing in the SC arrangement, which decreases the dislocation mobility significantly. Due to the anisotropy of dislocation line tension, the dislocation loops expand preferentially in those channels where their edge segments can move a longer distance. As soon as the motion of dislocations in these channels is hindered by the precipitates, a higher-level of stress is required for them to glide into alternative but non-preferential channels. Thus, both dislocation blockage by precipitates and line-tension anisotropy contribute to the increased hardening behaviour in BCC arrangement. Consequently, in the work of Pollock and Arogn (1992), Meissonnier et al. (2001) and Choi et al. (2005), the hardening rate and yielding stress were inevitably underestimated, as the precipitates were assumed to be in a perfect SC arrangement in their explicit unit-cell FE model. 
To examine the effect of the precipitate edge feature on the stress-strain response, DDD simulations were carried out considering precipitates with sharp and round edges (Choi et al., 2005). The channel width and the precipitate size were chosen to be $250 \mathrm{~b}$ and $1500 \mathrm{~b}$, respectively. The edge radius for the roundly edged precipitate case was 200b, corresponding to a precipitate volume fraction of $61 \%$. The simulation results show that the stress-strain responses are very similar for these two edge geometries, indicating a weak influence of precipitate edge feature. This differs from the findings of Choi et al. (2005), who predicted that a change of the radius of the $\gamma^{\prime}$ precipitate edge can influence the constraint on plastic flow significantly, resulting in drastic changes in the stress-strain curves, especially for the softening stage. However, the present DDD simulations show that the most important constraint effect on dislocation motion comes from the channel width. A small modification of the precipitate edge geometry does not change the channel width significantly, hence influence little the dislocation mobility or the associated plastic flow within the channels.

\subsection{Effect of lattice misfit}

For nickel alloys, the lattice mismatch between $\gamma$ and $\gamma^{\prime}$ phases gives rise to the misfit strain and the associated coherency stress in the material, which can be calculated by Eq.(5) and the results agree well with the FEM simulations (Ohashi et al., 1997). Following Brien et al. (2001), the coherency stress was considered as an internal stress field in the present simulations. The results in Fig.15 showed that, the coherency stress has negligible influence on the stress-strain behaviour for a precipitate size $l=1500 \mathrm{~b}$ and volume fraction $f=63 \%$. This may be explained as follows. When a dislocation overloops a precipitate under the [001] 
tension loading, the resolved shear stress on the slip plane from the misfit stress field can promote the dislocation motion in the [001] horizontal channel but obstruct the dislocation motion in one of the two vertical channels. The promotional effect and the obstructive effect cancel out each other and consequently no influence on the stress-strain response is introduced by the misfit stress field. The present simulation follows the work of Brien et al. (2001) by assuming that the coherency stress was distributed uniformly both in the matrix phase and in the precipitate. In Rao et al.'s DDD simulation (2004), a non-uniform coherency stress suggested by Duesbery et al. (1992) was employed, where the coherency stress is largest at the interface and decays rapidly $\left(\sim r^{-5}\right)$ from the interface. They found that the non-uniform coherency stress can enhance the critical resolved shear stress (CRSS) to a certain extent depending on the APB energy. However, the study was carried out for spherical precipitates. For cubic precipitates, effects of the non-uniform coherency stress on the mechanical response need to be further investigated. Furthermore, the lattice misfit introduces not only the coherency stress but also the intrinsic misfit dislocation around the $\gamma / \gamma^{\prime}$ interface. These intrinsic misfit dislocations knocking out the long-range coherency stress, which are formed before loading but not considered in the present DDD simulation, could interact with dislocations approaching the interface and impede them from shearing into the precipitate and thus contribute to the hardening effect. Although the coherency stress has negligible effect on the stress-strain response, the lattice misfit between two phases can still strengthen the superalloy by the formed intrinsic misfit dislocations.

\subsection{Simulation of cyclic deformation}

Some strain-controlled cyclic responses were modelled for the RVC using the DDD method. The cyclic loading, with a triangular waveform, was applied in the [001] direction at a strain 
rate of $0.001 \mathrm{~s}^{-1}$ and a strain range of $2 \%$. For the purpose of comparison, the precipitate size and channel width are selected to be $1920 b \times 2000 b \times 2080 b$ and $250 \mathrm{~b}$, respectively, which are the typical values for CMSX4 (Tinga et al., 2010). Due to computing limitation, only the first two cycles were simulated and shown in Fig.16, where the simulated stress-strain loops are compared with the stabilised loops of CMSX-4 obtained experimentally by Vattre (2009). It can be seen from Fig.16 that there is little cyclic hardening or softening for the material, consistent with the experimental observation by Vattre et al. (2009), and the simulated stress-strain loops of the first two cycles fit well with the stabilised hysteresis loop in Vattre (2009). It is recognised that cyclic hardening or softening is associated with the precipitate shearing and the formation of irreversible dislocation structure. Cross slip plays an important role in the formation of the irreversible dislocation structures such as the persistent slip band (PSB) (Shin et al., 2007). In the present DDD simulation, for the applied loading level and direction, cross slip is hard to occur (Tinga et al., 2010) and this is also true for precipitate shearing by superdislocations. Thus the dislocation motions are mostly reversible and no cyclic hardening or softening is obtained. This can be verified from the simulated dislocation density evolution, which shows that the dislocation density can decrease to a value close to the initial dislocation density due to the reversible motion of most dislocations.

In the present paper, the mechanical behaviors of single crystal nickel-based superalloy were modeled by the DDD method. It would be ideal to carry out experimental observations for validation of the present results. For instance, to validate the connection between the 3stage stress-strain responses and dislocation precipitate shearing, it is desirable to have an in-situ TEM observation of the dislocation evolution in both the matrix and precipitates. Also, 
the TEM observations can be used to quantify the total dislocation density, junction density and jog density, which can be compared with the DDD simulation to validate the proposed hardening mechanism. The effect of the channel width and precipitate size on the stressstrain behavior and the dislocation density evolution could be validated from mechanical tests of materials processed with varying channel width and precipitates sizes. This information will provide invaluable guidance for further development of the DDD framework.

\section{Conclusions}

3D DDD simulations of mechanical deformation of single crystal Ni-based superalloys have been carried out by considering the interaction between dislocation and internal microstructure. Precipitate shearing by superdislocaitons is shown to be responsible for the softening behaviour of the material observed experimentally. The results also show that most of the dislocations are deposited on the two-phase boundaries and form a highly polarised network by dislocation reaction, which may be the main mechanism for the hardening behaviour of the material. Although the dislocation junctions and jogs, as well as the "V"-shaped configuration, can be found in the dislocation microstructure, their presence is too low to contribute significantly to the hardening behaviour.

For a fixed precipitate size, the smaller the channel width, the higher the hardening rate at the second stage and the higher the flow stress at the third stage. For a given channel width, the larger the precipitate size, the higher the hardening rate in the second stage and the higher the flow stress in the third stage. It is easier for dislocation pair to cut into smaller precipitates. For a given precipitate volume fraction, the scale-dependent hardening rate 
and the $0.2 \%$ proof yield stress at the second stage are mainly controlled by the channel width, i.e., increase with the decrease of the channel width. While, at the third softening stage, the channel width and the precipitate size both influence the flow stress.

The shape of the precipitate was found to affect the stress-strain response. For a given precipitate volume fraction, spherical precipitates tend to enhance the hardening rate and yield stress than cuboidal ones, due to the presence of the narrowest channel sections which block the motion of the dislocations. The precipitate arrangement also influences the mechanical behaviour, where the hardening rate and yield stress are higher for BCC arrangement than those for ideal SC arrangement. The coherency stress field caused by lattice misfit has a negligible effect on the stress-strain response, at least for the cuboidal precipitates considered in this work.

Preliminary cyclic simulations were also carried out using the DDD model. No cyclic hardening or softening was found in the material due to the lack of precipitate shearing and dislocation cross slip, consistent with the experimental results of Vattre (2009).

\section{References}

1. Akarapu S., Zbib H.M., Bahr D.F., 2010. Analysis of heterogeneous deformation and dislocation dynamics in single crystal micropillars under compression. International Journal of Plasticity 26, 239-257.

2. Arsenlis A., Cai W., Tang M., Rhee M., Oppelstrup T., Hommes G., Pierce T.G. and Bulatov V.V., 2007. Enabling strain hardening simulations with dislocation dynamics. Modelling and Simulation in Materials Science and Engineering 15, 553-595 
3. Arsenlis, A., Parks, D.M., 1999. Crystallographic aspects of geometrically-necessary and statistically-stored dislocation density. Acta Meterialia 47, 1597-1611.

4. Brien, V., Kubin, L.P., Decamps, B., 2001. Low-cycle fatigue of a nickel-based superalloy at high temperature: Simplified micromechanical modelling. Philosophical Magazine A $81,2285-2301$

5. Busso, E.P., Meissonnier, F.T., O’Dowd, N.P., 2000. Gradient-dependent deformation of two-phase single crystals. Journal of the Mechanics and Physics of Solids 48, 2333-2361.

6. Choi, Y.S., Parthasarathy, T.A., Dimiduk, D.M., Uchic, M.D., 2005. Numerical study of the flow responses and the geometric constraint effects in Ni-base two-phase single crystals using strain gradient plasticity. Materials Science and Engineering A 397,69-83.

7. de Wit R. 1967. Some relations for straight dislocations. physica status solidi 20:567-573

8. Demura M., Golberg D., Hirano T., 2007. An athermal deformation model of the yield stress anomaly in Ni3Al. Intermetallics 15:1322-1331

9. Déprés, C., Robertson, C.F., Fivel, M.C., 2004. Low-strain fatigue in aisi 316l steel surface grains: A three-dimensional discrete dislocation dynamics modelling of the early cycles i. Dislocation microstructures and mechanical behaviour. Philosophical Magazine 84, 2257-2275

10. Déprés, C., Robertson, C.F., Fivel, M.C., 2006. Low-strain fatigue in 316L steel surface grains: A three dimension discrete dislocation dynamics modelling of the early cycles. Part 2: Persistent slip markings and micro-crack nucleation. Philosophical Magazine 86, 79-97.

11. Devincre B., Kubin L. P., Lemarchand C., Madec R., 2001. Mesoscopic simulations of plastic deformation. Materials Science and Engineering A 309-310, 211-219.

12. Devincre, B., Veyssière, P., Kubin, L.P., Saada, G., 1997. A simulation of dislocation 
dynamics and of the flow stress anomaly in L12 alloys. Philosophical Magazine A 75, 1263-1286.

13. Devincre, B., Veyssière, P., Saada, G., 1999. Simulation of the plastic flow in $\mathrm{Ni}_{3} \mathrm{Al}$ : Work hardening and strain-rate sensitivity. Philosophical Magazine A 79, 1609-1627.

14. Devincre, B., Kubin, L., Hoc, T., 2007. Collinear superjogs and the low-stress response of fcc crystals. Scripta Materialia 57, 905-908.

15. Fedelich, B., 1999. A microstructure based constitutive model for the mechanical behavior at high temperatures of nickel-base single crystal superalloys. Computational Materials Science 16, 248-258.

16. Fedelich, B., 2002. A microstructural model for the monotonic and the cyclic mechanical behavior of single crystals of superalloys at high temperatures. International Journal of Plasticity $18,1-49$.

17. Groh S., Devincre B., Kubin L.P., Roos A., Feyel F., Chaboche J.-L., 2005. Size effects in metal matrix composites. Materials Science and Engineering A 400-401, 279-282.

18. Gao Y., Zhuang Z., Liu Z.L., You X.C., Zhao X.C. and Zhang Z.H., 2010. Investigations of pipe-diffusion-based dislocation climb by discrete dislocation dynamics. International Journal of Plasticity doi:10.1016/j.ijplas.2010.11.003.

19. Ghoniem N.M., Tong S.H., Sun L.Z., 2000. Parametric dislocation dynamics: a thermodynamics-based approach to investigations of mesoscopic plastic deformation. Phys. Rev. B 61:913.

20. Ghoniem N.M., Huang J., Wang Z., 2002. Affine covariant-contravariant vector forms for the elastic field of parametric dislocations in isotropic crystals, Philos. Mag. Lett. 82:55-63.

21. Hirth J.P. and Lothe J., 1992. Theory of Dislocations, 2nd Edition, Krieger, Melbourne. 
22. Kear, B.H., Wilsdorf, G.F., 1962. Dislocation configurations in plastically deformed polycrystalline $\mathrm{Cu}_{3} \mathrm{Au}$ alloys. Transactions of the Metallurgical Society of the AIME 224, $382-386$.

23. Kubin L. P., Canova G., Condat M., Devincre B., Pontikis V. and Brechet Y., 1992 Dislocation microstructures and plastic flow: a 3D simulation. Solid State Phenom. 23-24: 455

24. Kubin L. P. and Devincre B., 1999. From dislocation mechanisms to dislocation microstructures and strain hardening. Deformation-Induced Microstructures: Analysis and Relation to Properties Ed. Bilde-Sorensen J. B. et al., Proc. 20th Int. Symp. on Materials Science (Roskilde, Denmark, 1999): 61-83

25. Liu Z.L., Liu X.M., Zhuang Z., You X.C., 2009. A multi-scale computational model of crystal plasticity at submicron-to-nanometer scales. International Journal of Plasticity 25 , 1436-1455.

26. Madec R., Devincre B., Kubin L.P., 2002. On the nature of attractive dislocation crossed states. Computational Materials Science 23: 219-224

27. Madec R., Devincre B., Kubin L., Hoc T. and Rodney D., 2003. The Role of Collinear Interaction in Dislocation-Induced Hardening. Science, 301: 1879-1882

28. Madec, R., Devincre, B., Kubin, L.P., 2003. In: Shibutani, Y., Kitagawa, H., editors. Mesoscopic dynamics in fracture process and strength of materials. Dordrecht: Kluwer, 115-135.

29. Madec R. and Kubin L., 2008. Second-order junctions and strain hardening in bcc and fcc crystals. Scripta Materialia, 58:767-770

30. McHugh, P.E. and Mohrmann, R., 1997. Modelling of creep in a Ni base superalloy using a single crystal plasticity model. Computational Materials Science 9(1-2), 134-140. 
31. Meissonnier, F.T., Busso, E.P., O'Dowd, N.P. 2001. Finite element implementation of a generalised non-local rate-dependent crystallographic formulation for finite strains. International Journal of Plasticity 17, 601-640.

32. Motz C., Weygand D., Senger J., Gumbsch P., 2008. Micro-bending tests: A comparison between three-dimensional discrete dislocation dynamics simulations and experiments. Acta Materialia 56, 1942-1955.

33. Ohashi, T., Hidaga, K., Saito, M., 1997. Quantitative study of the plastic slip deformation and formation of internal stresses in Ni-base superalloys. Materials Science \& Engineering A A238, 42-49.

34. Pollock, T.M., Argon, A.S., 1992. Creep resistance of CMSX-3 nickel base superalloy single crystals. Acta Metallurgica et Materialia 40, 1-30.

35. Queyreau S., Monnet G., Devincre B., 2009. Slip systems interactions in a-iron determined by dislocation dynamics simulations. International Journal of Plasticity $25,361-377$

36. Rao, S.I., Parthasarathy, T.A., Dimiduk, D.M., Hazzledine, P.M., 2004. Discrete dislocation simulations of precipitation hardening in superalloys. Philosophical Magazine 84, 3195-3215.

37. Rhee, M., Zbib, H.M., Hirth, J.P., H. Huang and T. D. de La Rubia, 1998. Models for Long/Short Range Interactions in 3D Dislocatoin Simulation. Modelling and Simulation in Materials Science and Engineering 6, 467-492.

38. Shenoy, M.M., Gordon, A.P., McDowell, D.L., Neu, R.W., 2005. Thermomechanical fatigue behavior of a directionally solidified Ni-base superalloy. Transactions of the ASME, Journal of Engineering Materials and Technology, ASME, USA 127, 325-337.

39. Shin, C.S., Robertson, C.F., Fivel, M.C., 2007. Fatigue in precipitation hardened materials: 
A three-dimensional discrete dislocation dynamics modelling of the early cycles. Philosophical Magazine 87, 3657-3669.

40. Sun, J., Lee, C.S., Lai, J.K.L., Wu, J.S., 1999. Dislocation dissociations and fault energies in Ni3Al alloys doped with palladium. Intermetallics 7, 1329-1335.

41. Tian, S., Zhou, H., Zhang, J., Yang H., Xu Y., Hu Z., 2000. Formation and role of dislocation networks during high temperature creep of a single crystal nickel-base superalloy Original Research Article Materials Science and Engineering A 279, 160-165

42. Tinga, T., Brekelmans, W. A. M., Geers, M. G. D., 2010. Cube slip and non-Schmid effects in single crystal Ni-base superalloys. Modelling and Simulation in Materials Science and Engineering 18, art. no. 015005.

43. Van der Giessen E. and Needleman A., Discrete dislocation plasticity: a simple planar model. Modelling and Simulation in Materials Science and Engineering 3:698-735

44. Vattré, A., Devincre, B., Roos, A., 2009. Dislocation dynamics simulations of precipitation hardening in $\mathrm{Ni}$-based superalloys with high $\gamma^{\prime}$ volume fraction. Intermetallics 17, 988-994.

45. Vattré, A., 2009. Strength of single crystal superalloys: from dislocation mechanisms to continuum micromechanics. PhD These. ONERA The French Aerospace Lab, pp.155.

46. Vattré A., Devincre B., Roos A., 2010. Orientation dependence of plastic deformation in nickel-based single crystal superalloys: Discrete-continuous model simulations. Acta Materialia 58, 1938-1951.

47. Veyssiere, P., Saada, G., 1997. Microscopy and plasticity of the L12 $\gamma^{\prime}$ phase. In: Dislocations in solids 10, 253.

48. Wang, L.N., Liu, Y., Yu, J.J., Xu, Y., Sun, X.F., Guan, H.R., Hu, Z.Q., 2009. Orientation and temperature dependence of yielding and deformation behavior of a nickel-base single 
crystal superalloy. Materials Science and Engineering A 505, 144-150.

49. Wang Z.Q., Ghoniem N. M., Swaminarayan S. and LeSar R., 2006. A parallel algorithm for 3D dislocation dynamics. Journal of Computational Physics 219:608-621

50. Wang Z.Q., Beyerlein I.J. and LeSar R., 2007. The importance of cross-slip in high-rate deformation. Modelling and Simulation in Materials Science and Engineering 15:675690

51. Wang Z.Q., Beyerlein I.J., LeSar R., 2008. Slip band formation and mobile dislocation density evolution in high-rate deformation of single fcc crystals. Philosophical Magazine 88:1321- 1343

52. Wang Z.Q., Beyerlein I.J., LeSar R., 2009. Plastic anisotropy in fcc single crystals in high rate deformation. International Journal of Plasticity 25, 26-48

53. Wang Z.Q. and Beyerlein I.J., 2010. An atomistically-informed dislocation dynamics model for the plastic anisotropy and tension-compression asymmetry of BCC metals. International Journal of Plasticity. 10.1016/j.ijplas.2010.08.011.

54. Yashiro K., Kurose F., Nakashima Y., Kubo K., Tomita Y., Zbib H.M., 2006. Discrete dislocation dynamics simulation of cutting of $\gamma^{7}$ precipitate and interfacial dislocation network in Ni-based superalloys. International Journal of Plasticity 22,713-723.

55. Zbib, H.M., Rhee, M. and Hirth, J.P., 1998. On Plastic Deformation and the Dynamcis of 3D Dislocations. International Journal of Mechanical Sciences 40, 113-127.

56. Zbib, H.M., Diaz de la Rubia, T., 2002. A multiscale model of plasticity. International Journal of Plasticity 18, 1133-1163. 


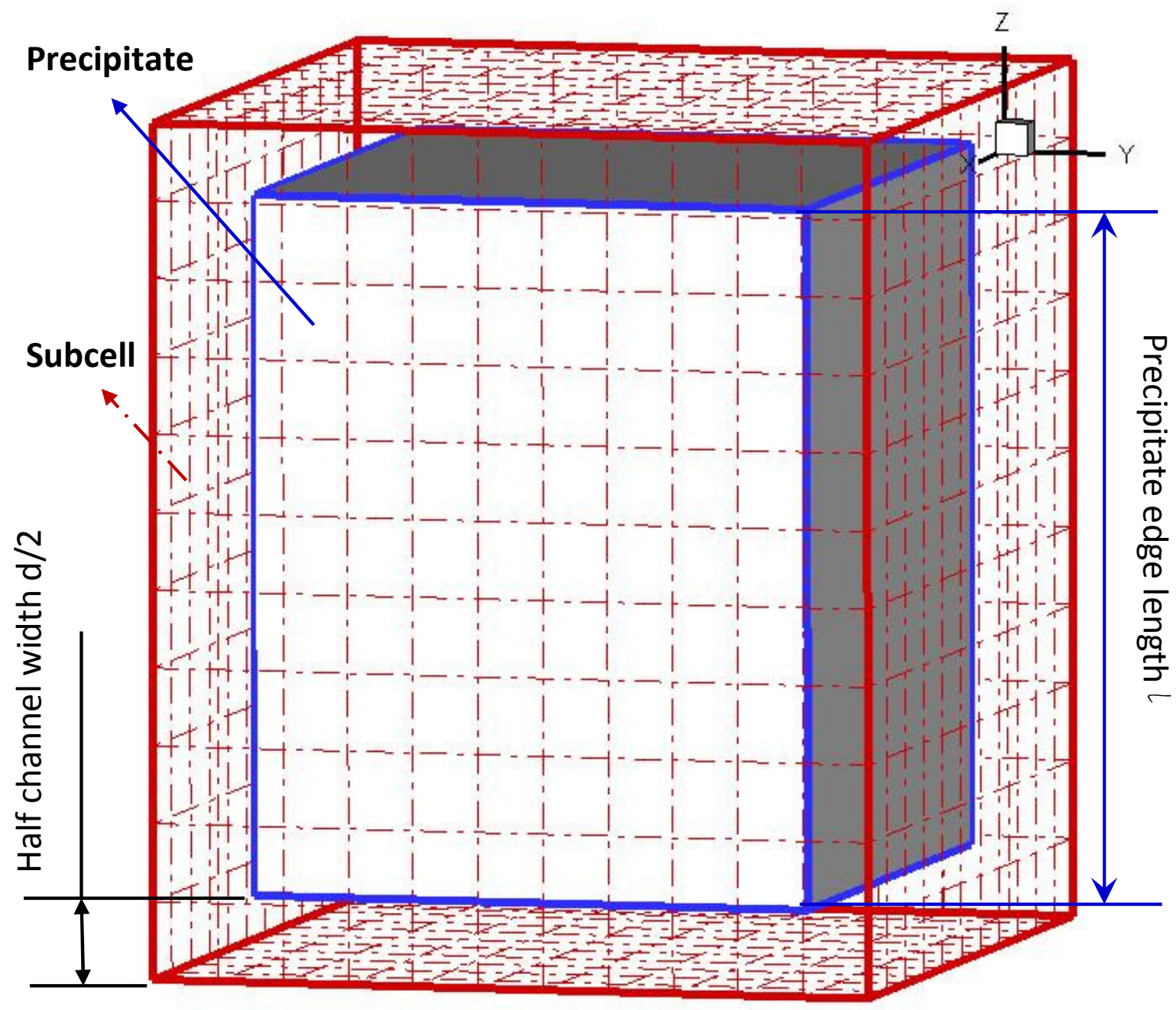

Fig. 1 The representative volume cell (RVC) comprising of $\gamma^{\prime}$ precipitate and $\gamma$ matrix. The RVC was divided into $12 \times 12 \times 12$ subcells. 


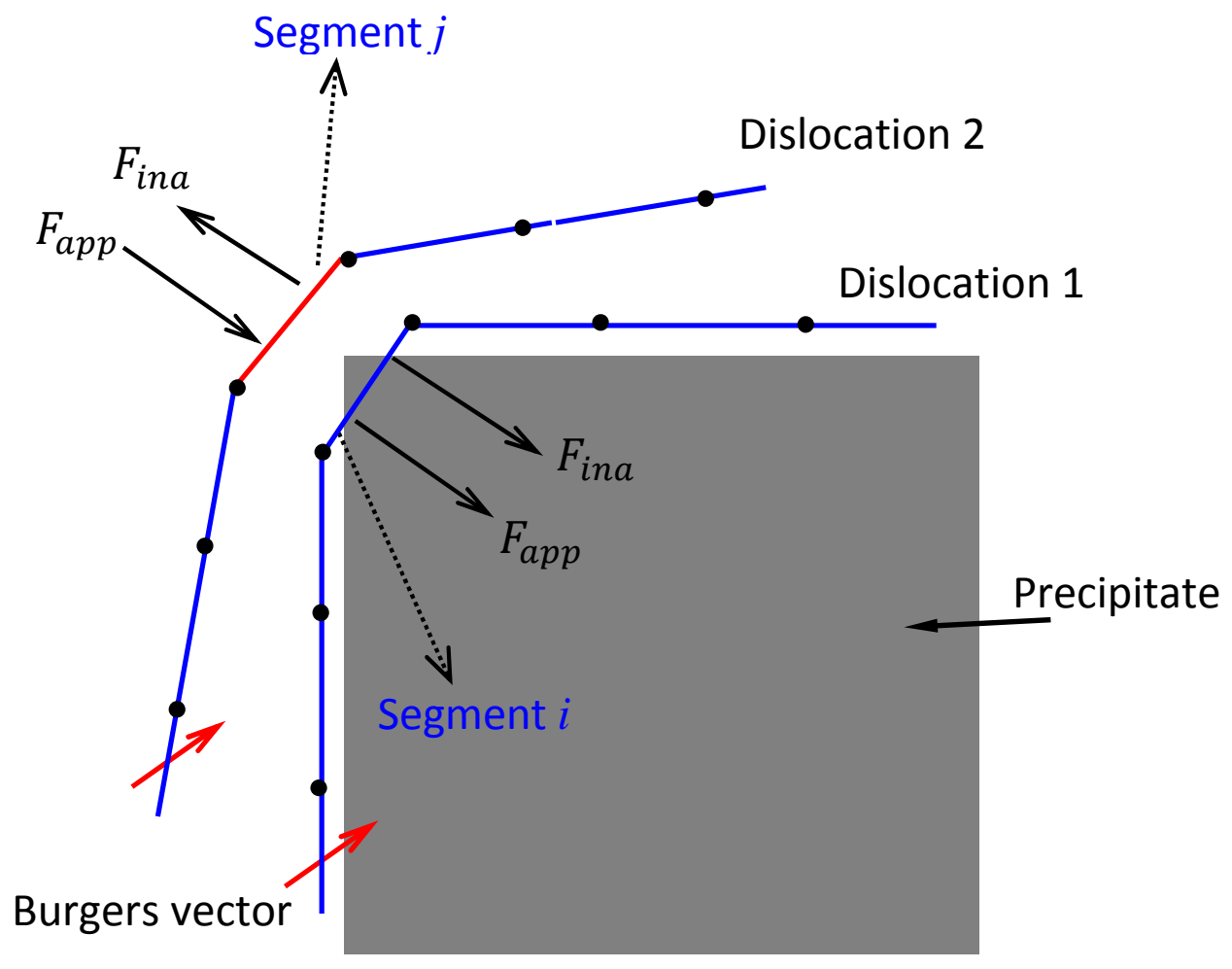

Fig. 2. Schematic of the interaction dislocation (segment $j$ ) of the segment $i$ and the corresponding forces acting on them. 


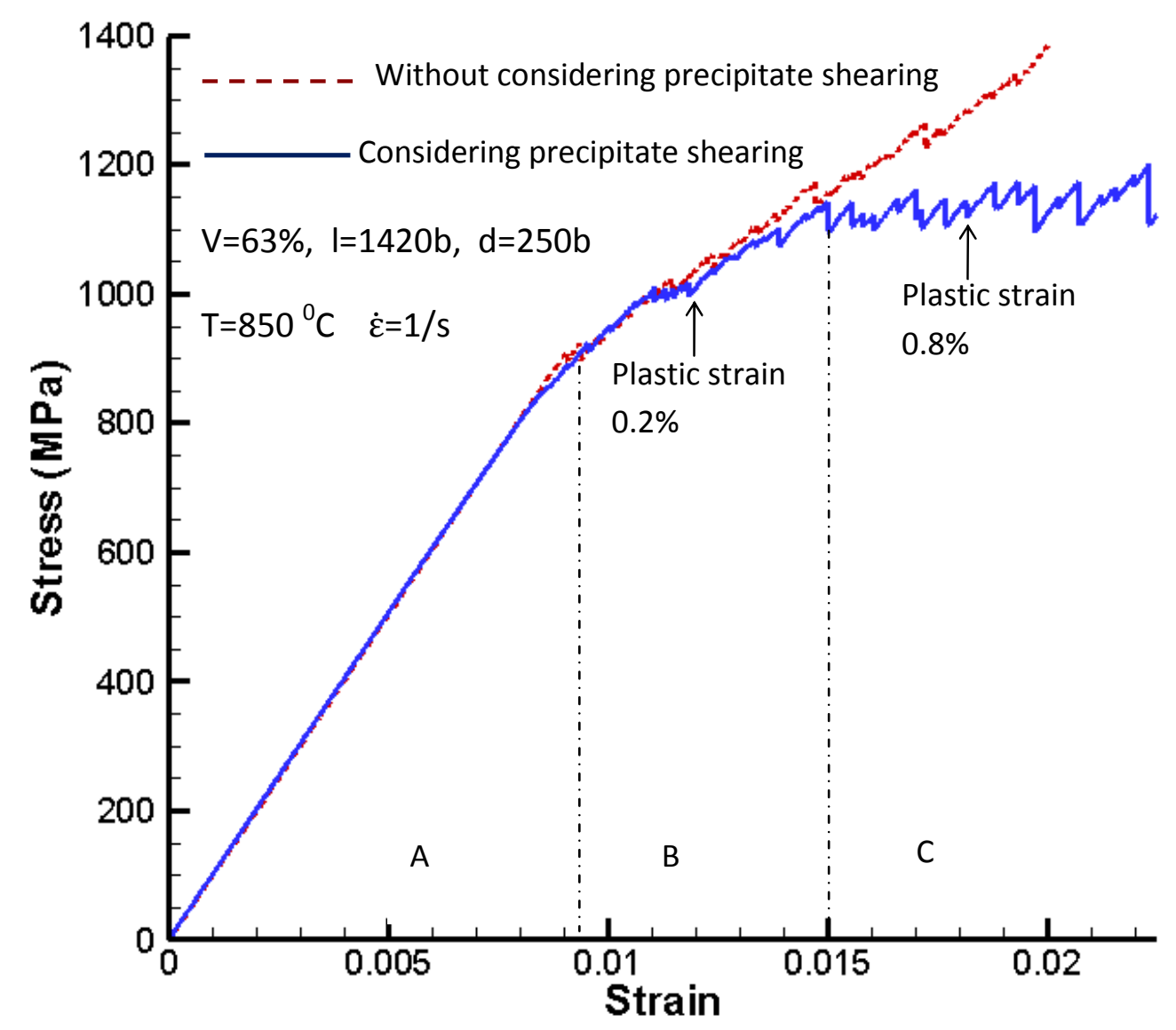

Fig 3. The stress-strain response with and without considering the precipitate shearing by superdislocations. 


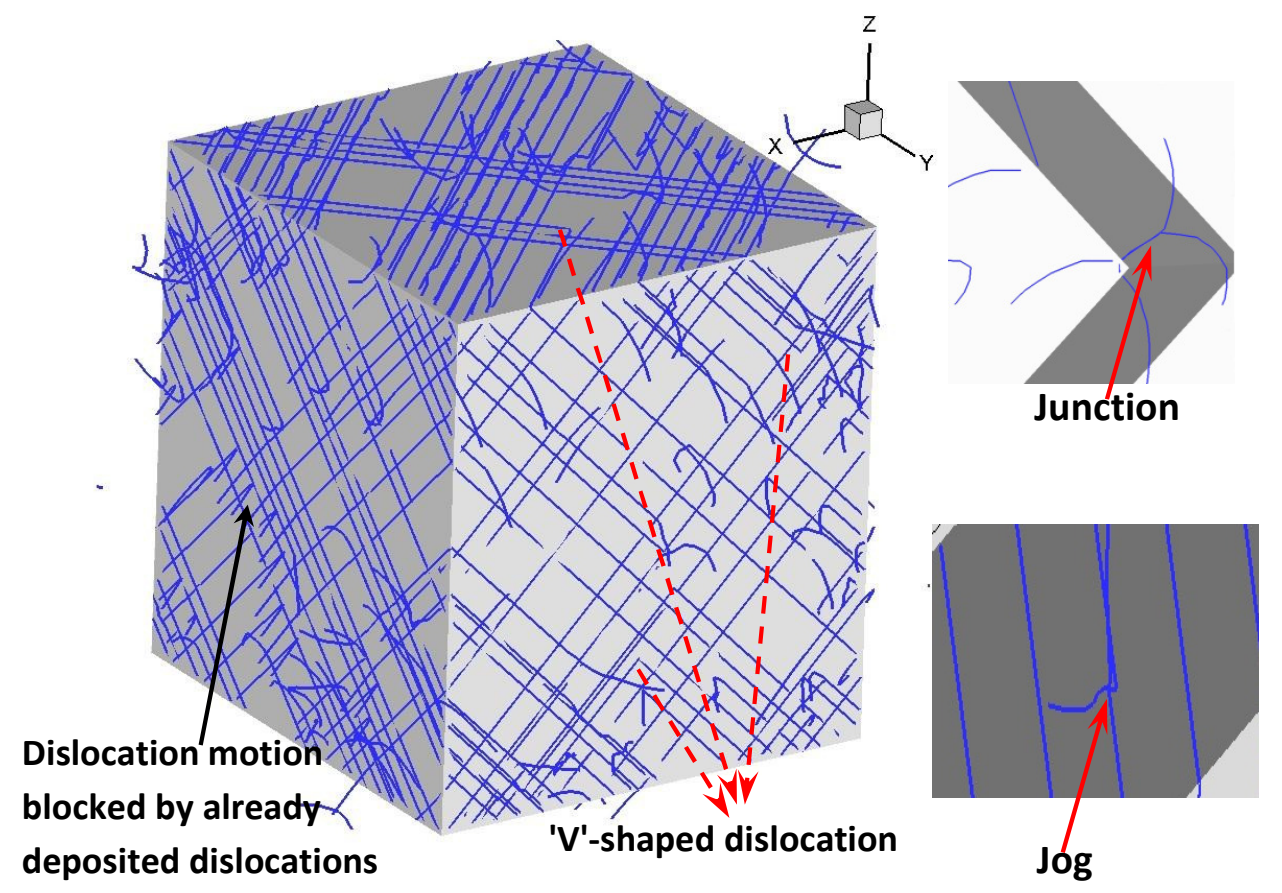

Fig 4. The dislocation microstructure at $0.2 \%$ plastic strain, which shows the dislocation network, junctions, jogs and ' $V$ '-shaped configuration. 


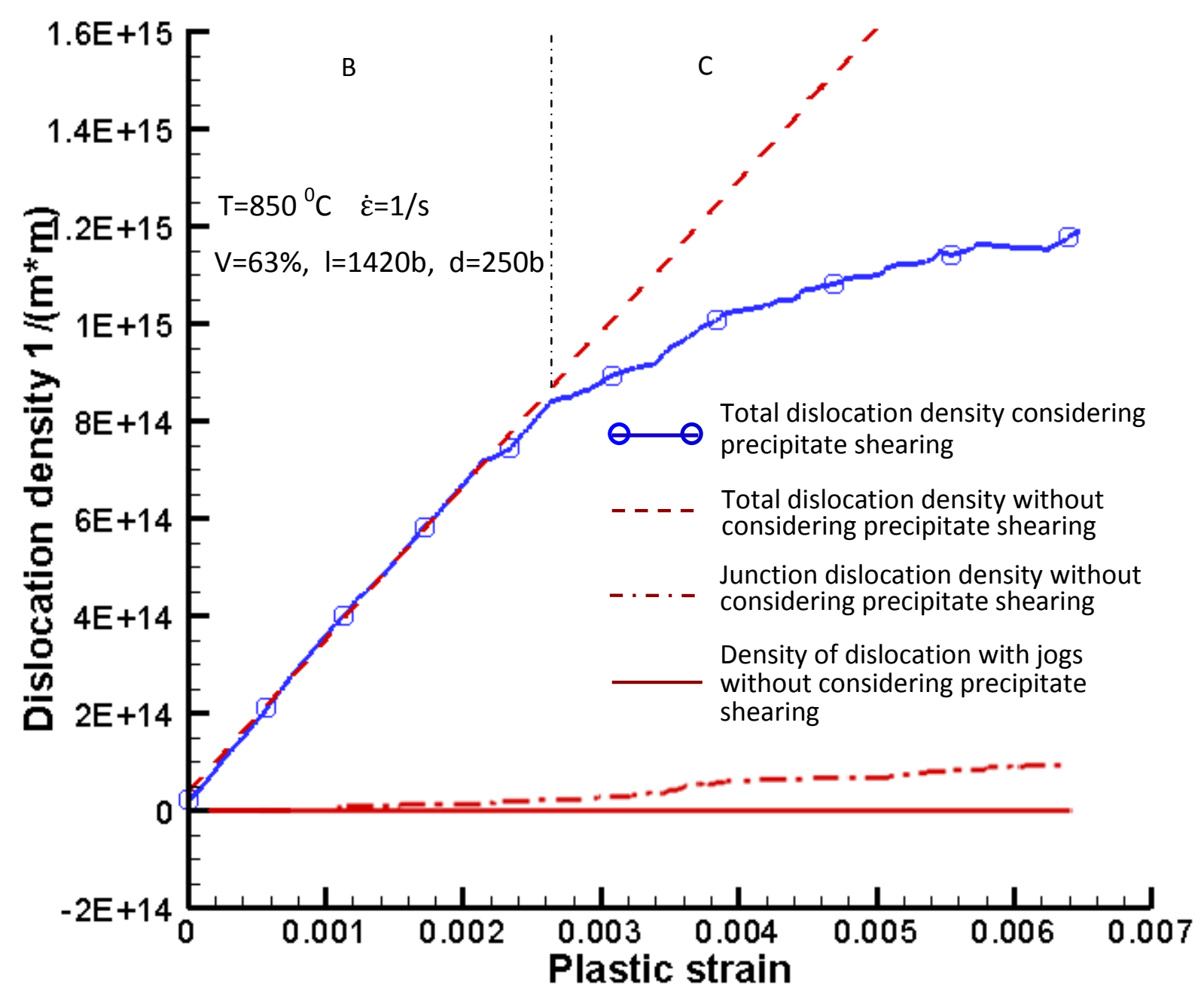

Fig 5. Evolution of the dislocation density as a funtion of the plastic strain with and without considering precipitate shearing by superdislocations. 


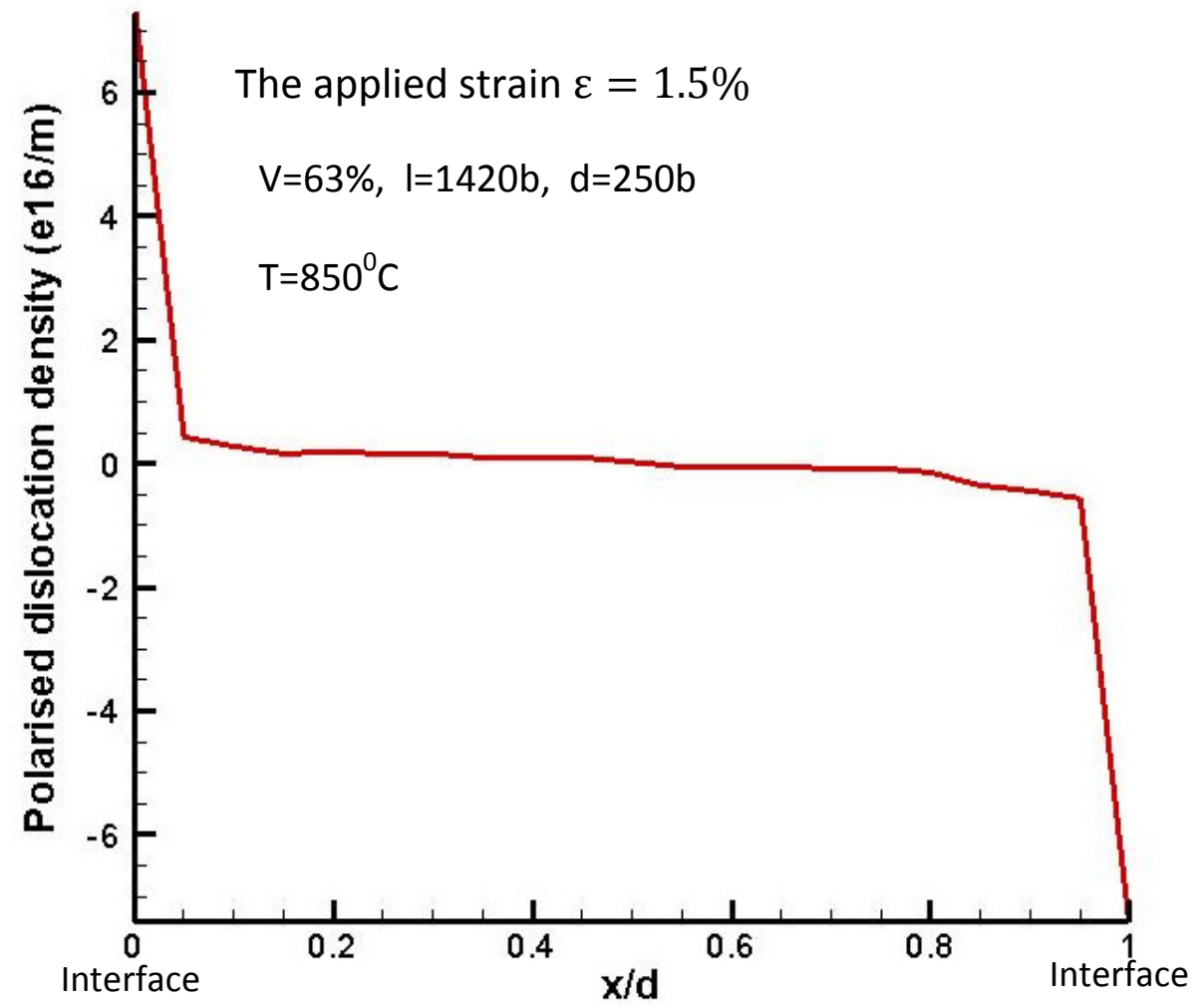

Fig 6. Density of the polarised dislocation against the normalized horizontal channel width $\mathrm{x} / \mathrm{d}$ at $1.5 \%$ strain. 


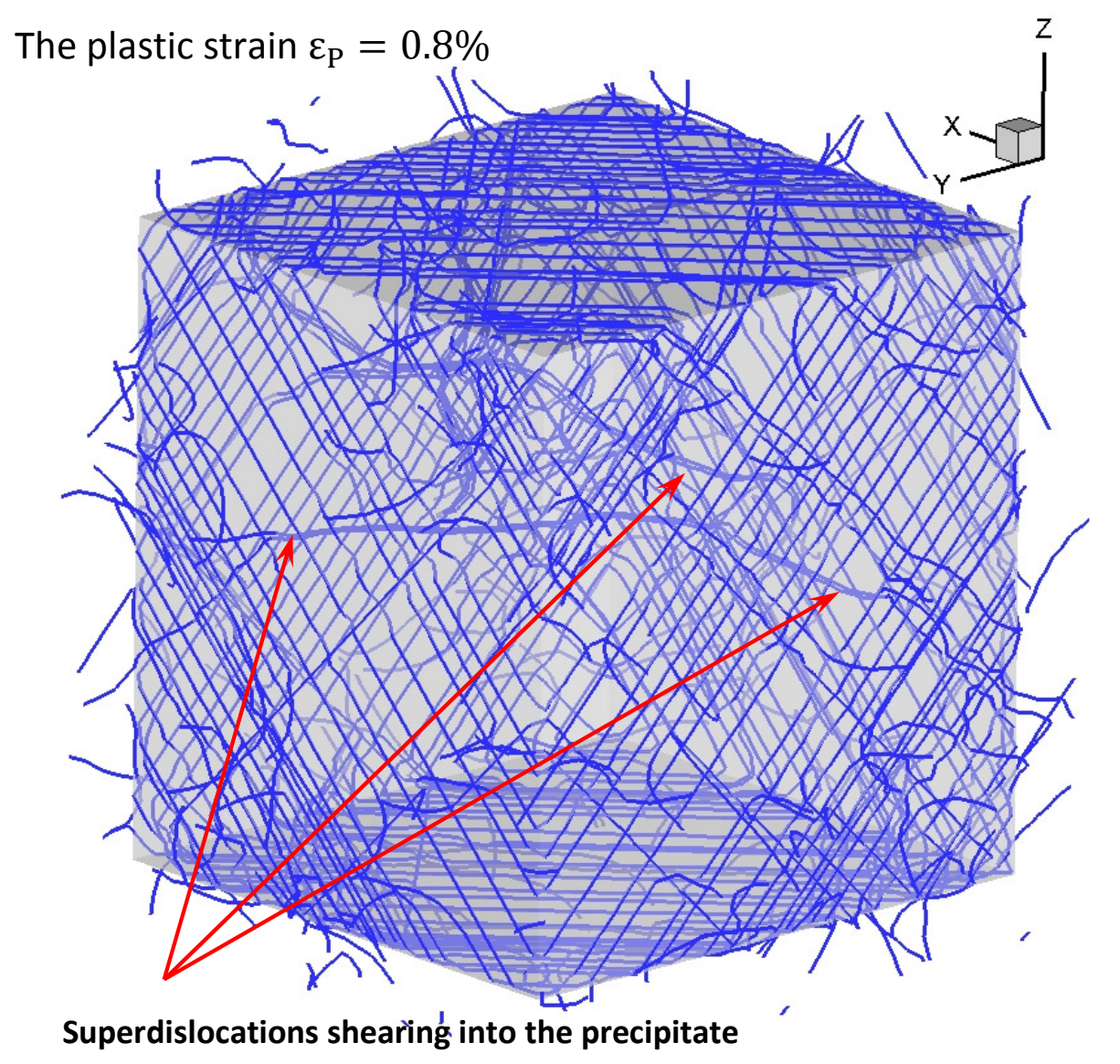

Fig.7 The dislocation microstructure at the $0.8 \%$ plastic strain, which shows a series of superdislocations cutting into the precipitate. 


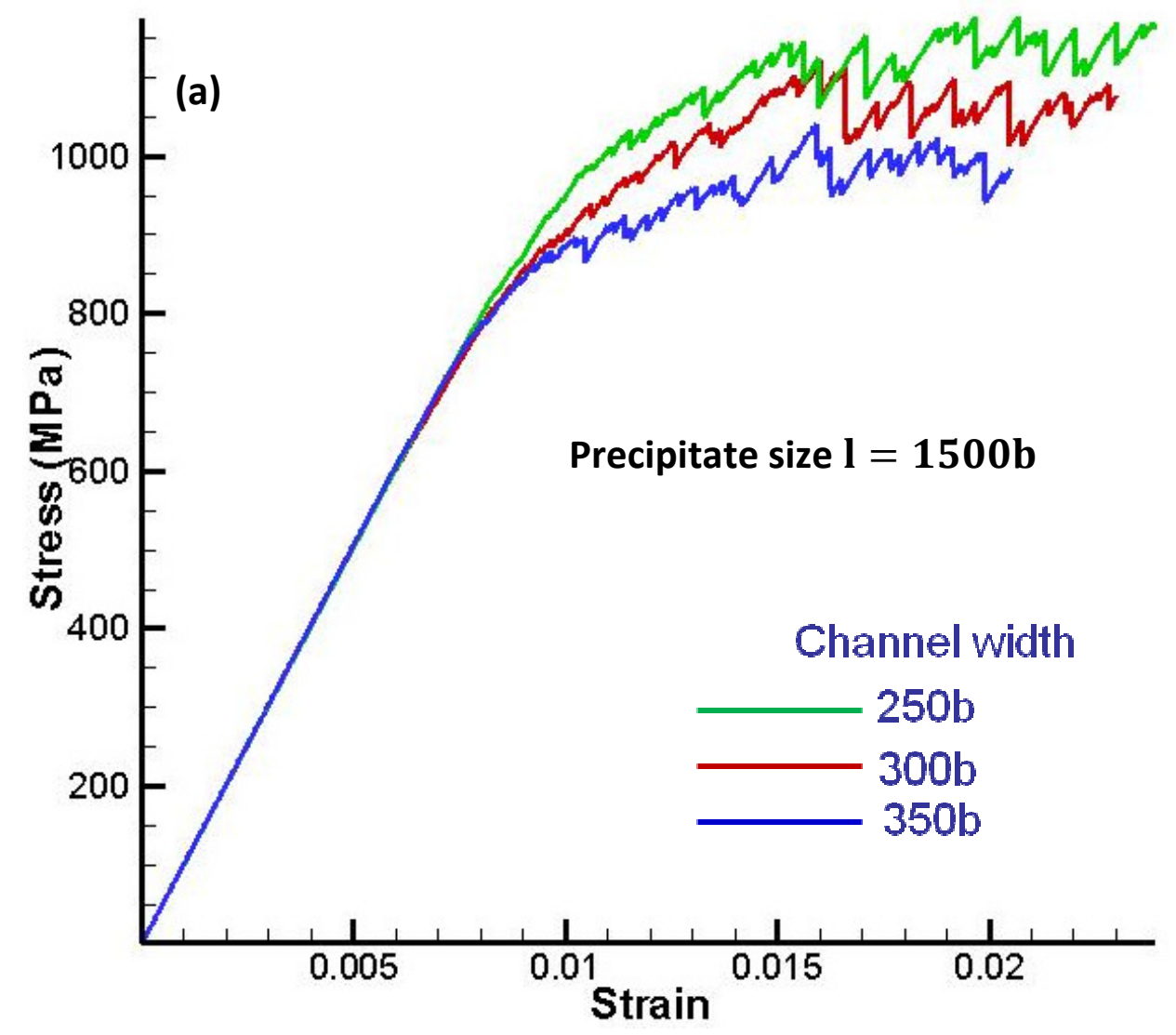

Fig.8 (a) The stress-strain response for three selected channel widths $d=\{250,300,350\}$ (b) at a fixed precipitate size of $1420 \mathrm{~b} \times 1500 \mathrm{~b} \times 1580 \mathrm{~b}$. 


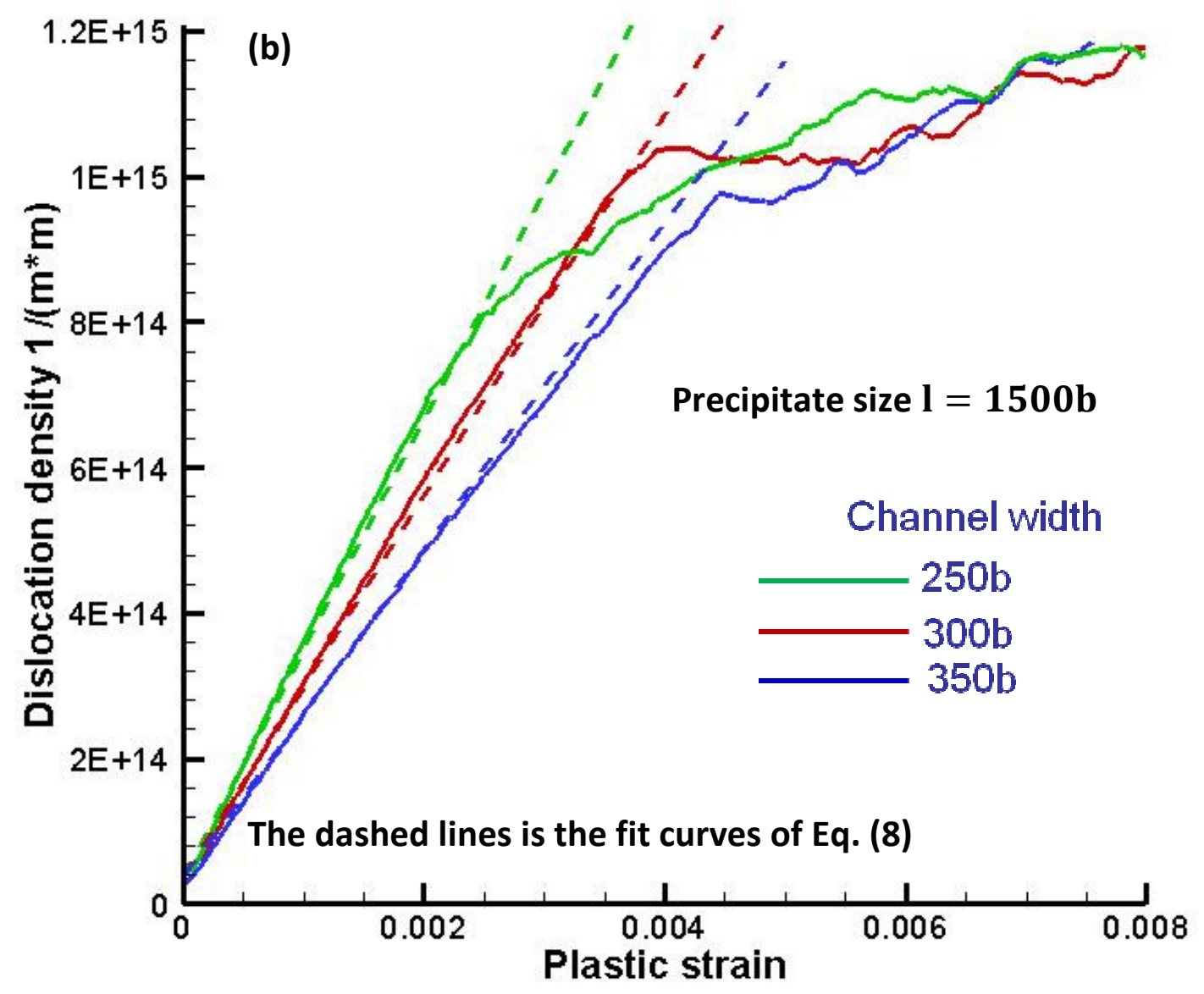

Fig.8(b) Evolution of dislocation density as a function of the plastic strain $\varepsilon_{p}$ for three selected channel widths $d=\{250,300,350\} \mathrm{b}$ at a fixed precipitate size of $1420 \mathrm{~b} \times 1500 \mathrm{~b} \times 1580 \mathrm{~b}$. 


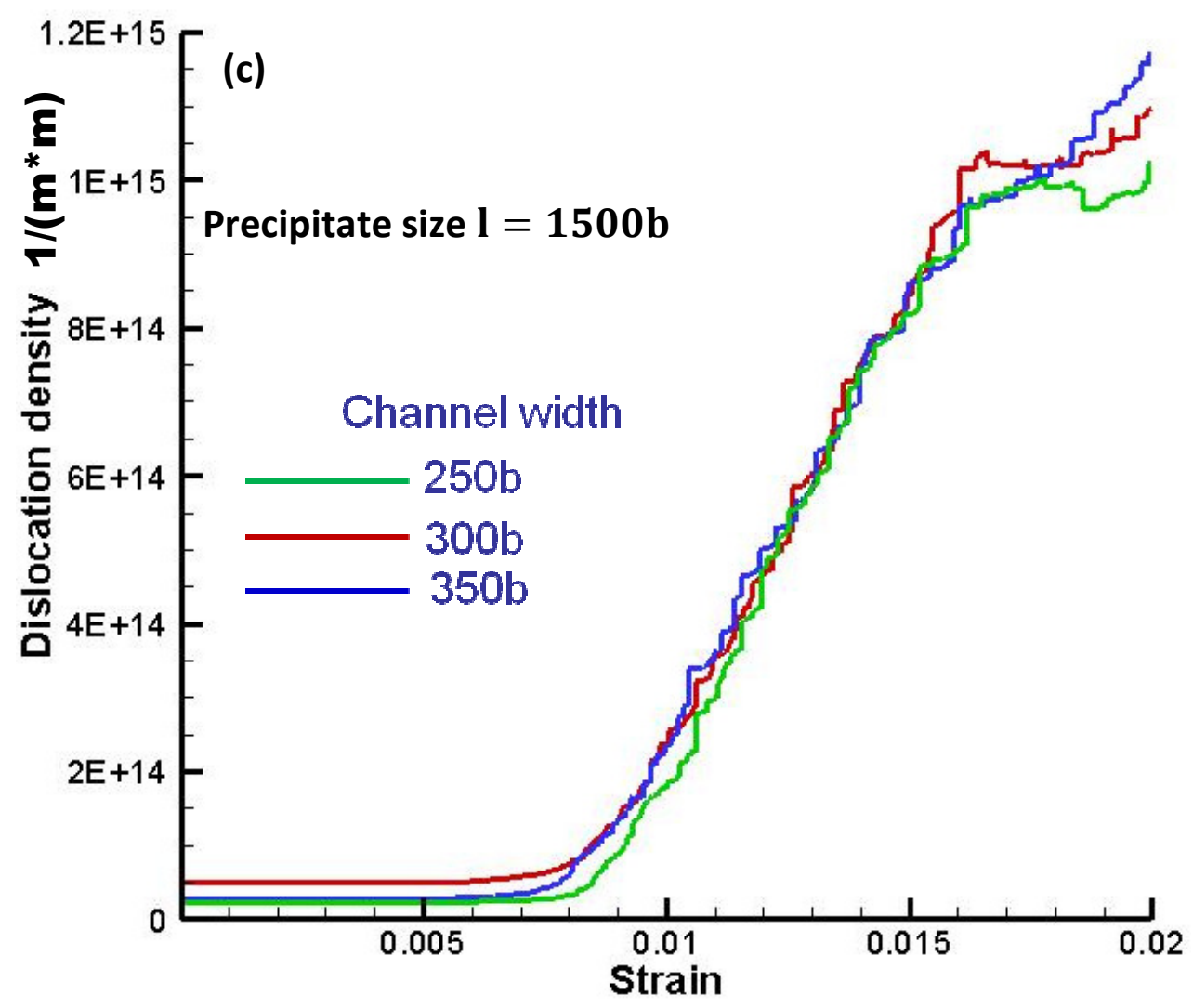

Fig.8 (c) Evolution of dislocation density as a function of the total applied strain $\varepsilon$ for three selected channel widths $d=\{250,300,350\} \mathrm{b}$ at a fixed precipitate size of $1420 \mathrm{~b} \times 1500 \mathrm{~b} \times 1580 \mathrm{~b}$. 


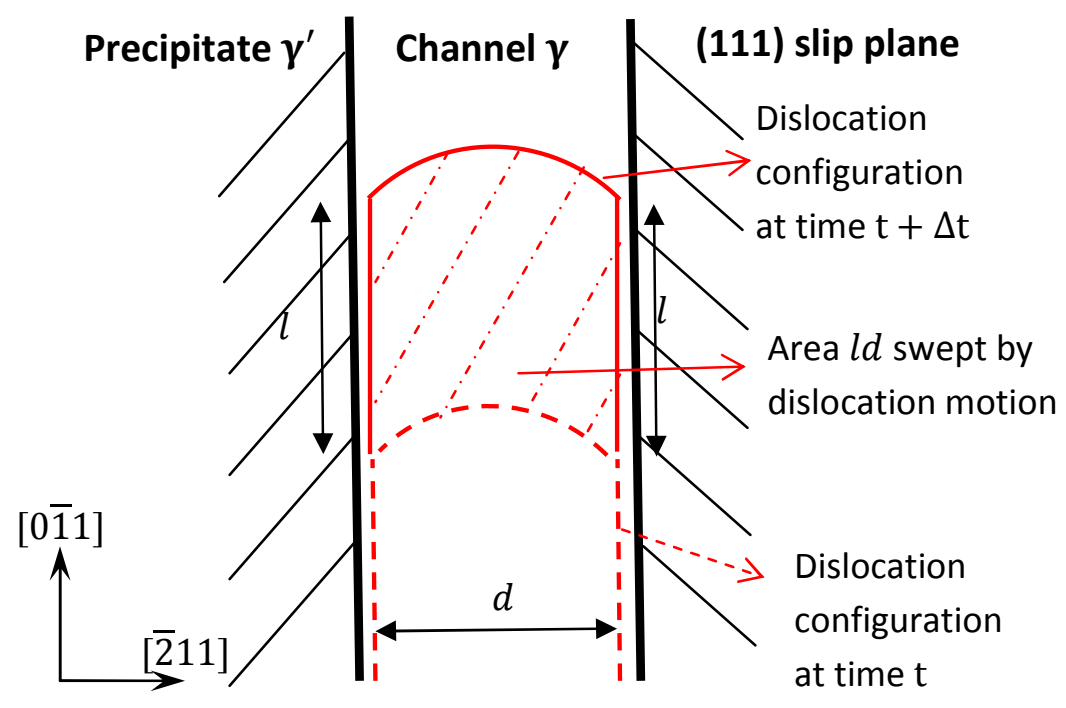

Fig.9 Schematic diagram of dislocation motion in the matrix channel during the time step $\Delta \mathrm{t}$. 


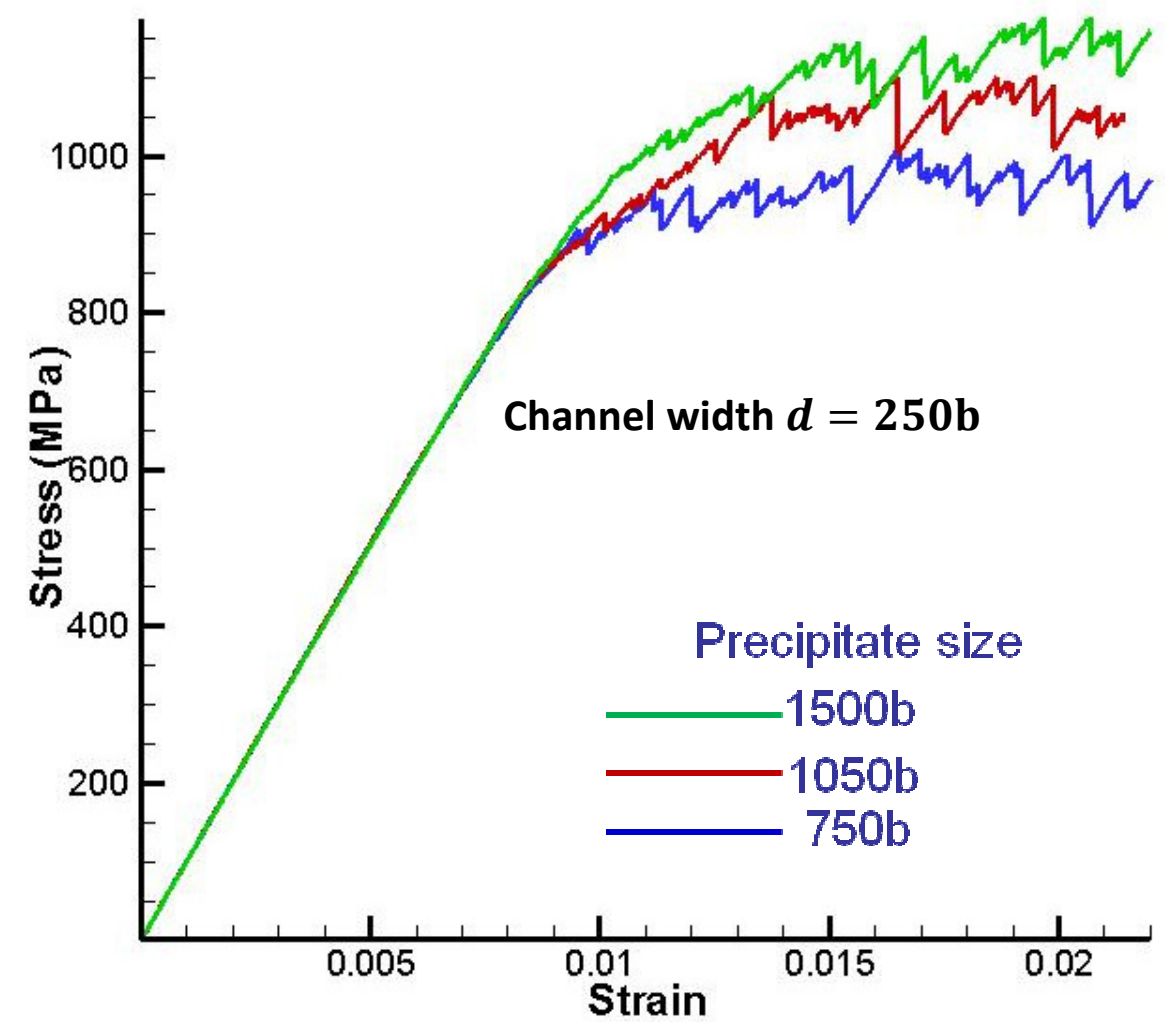

Fig.10 The stress-strain response for three selected precipitate sizes $l=\{750,1050,1420\} b$ at a constant channel width $d=250 b$. 


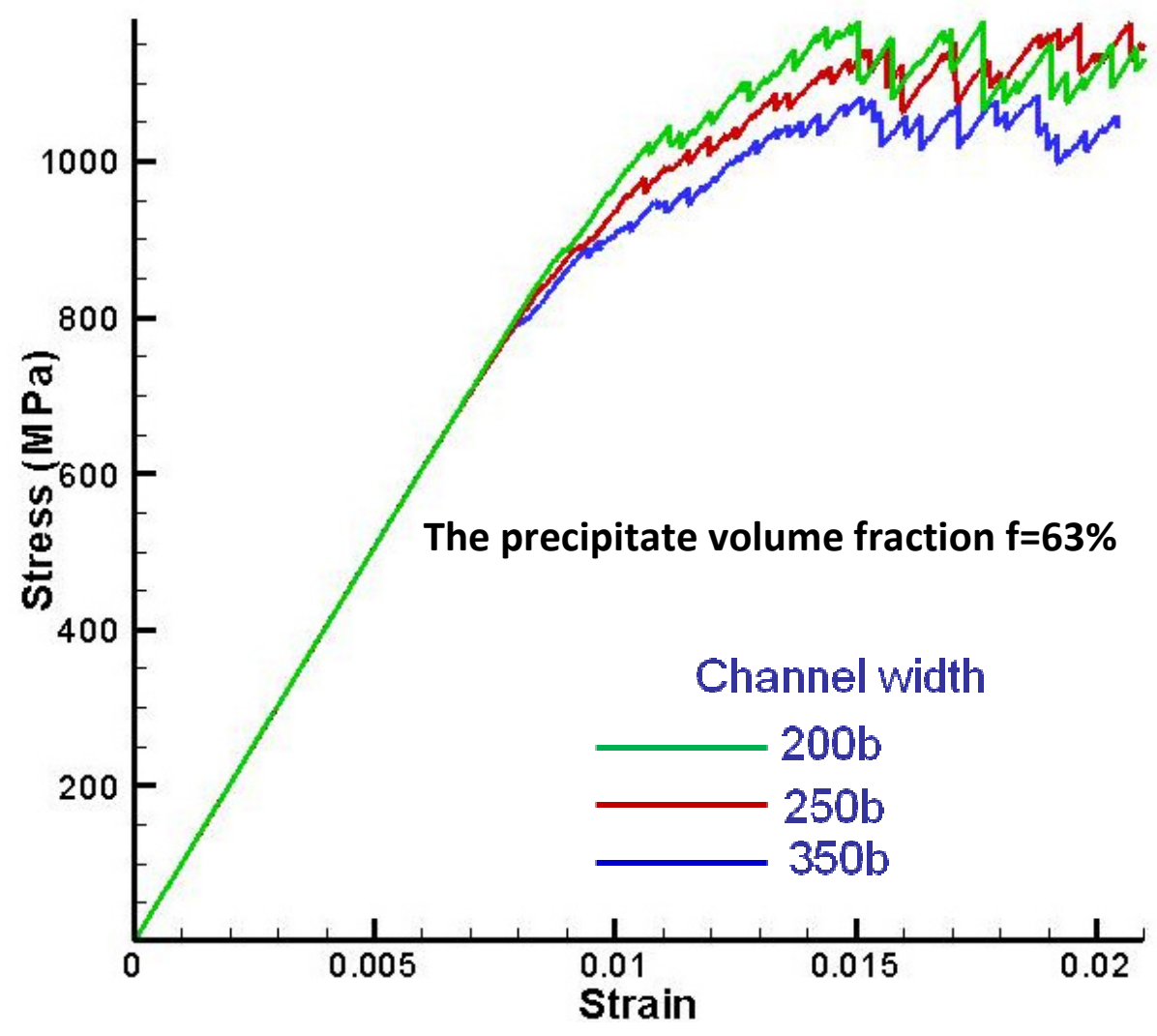

Fig. 11 The stress-stress response for three different RVC sizes for a fixed precipitate volume fraction $63 \%$. 


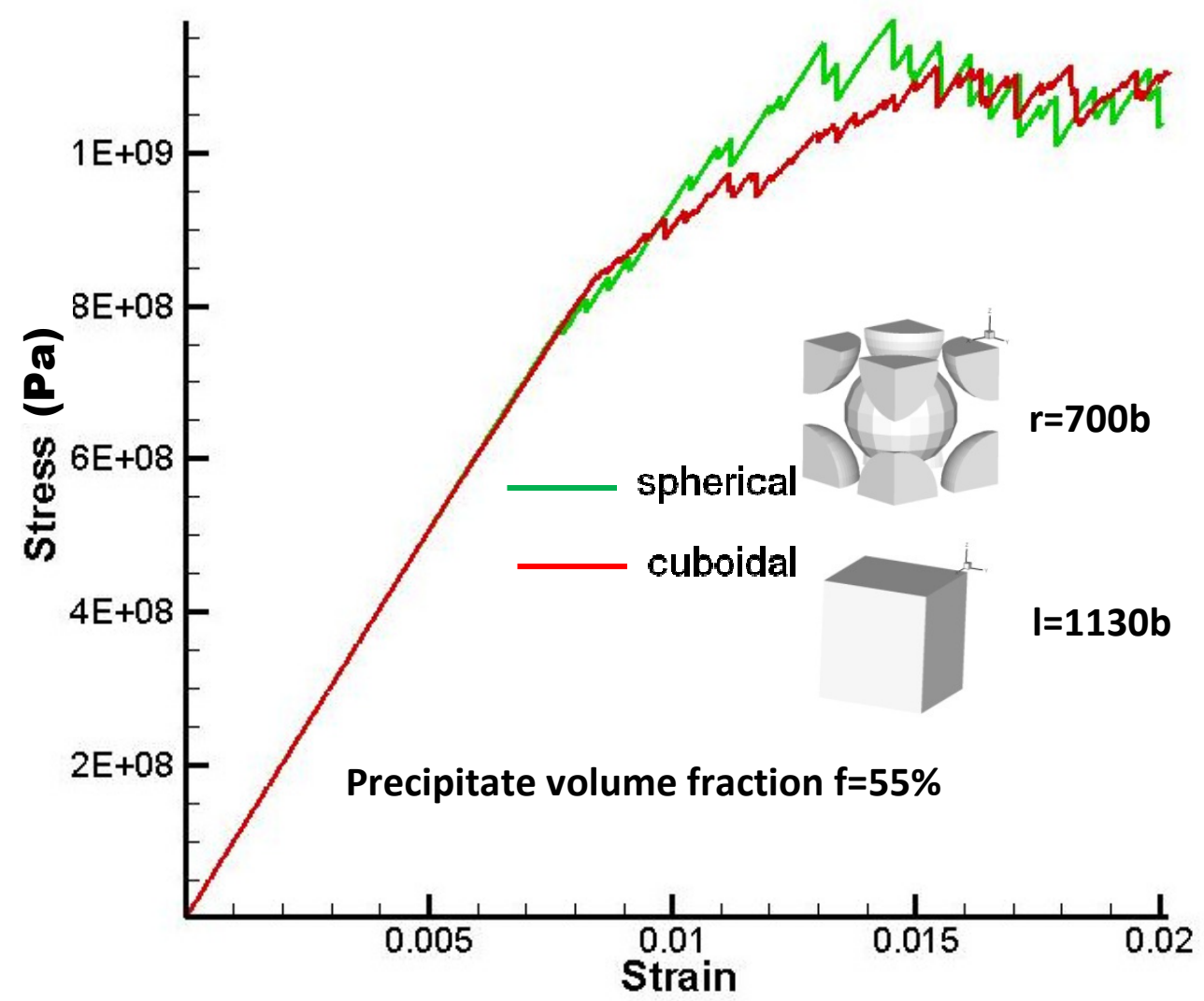

Fig. 12 Effect of precipitate shape on the stress-strain response. 


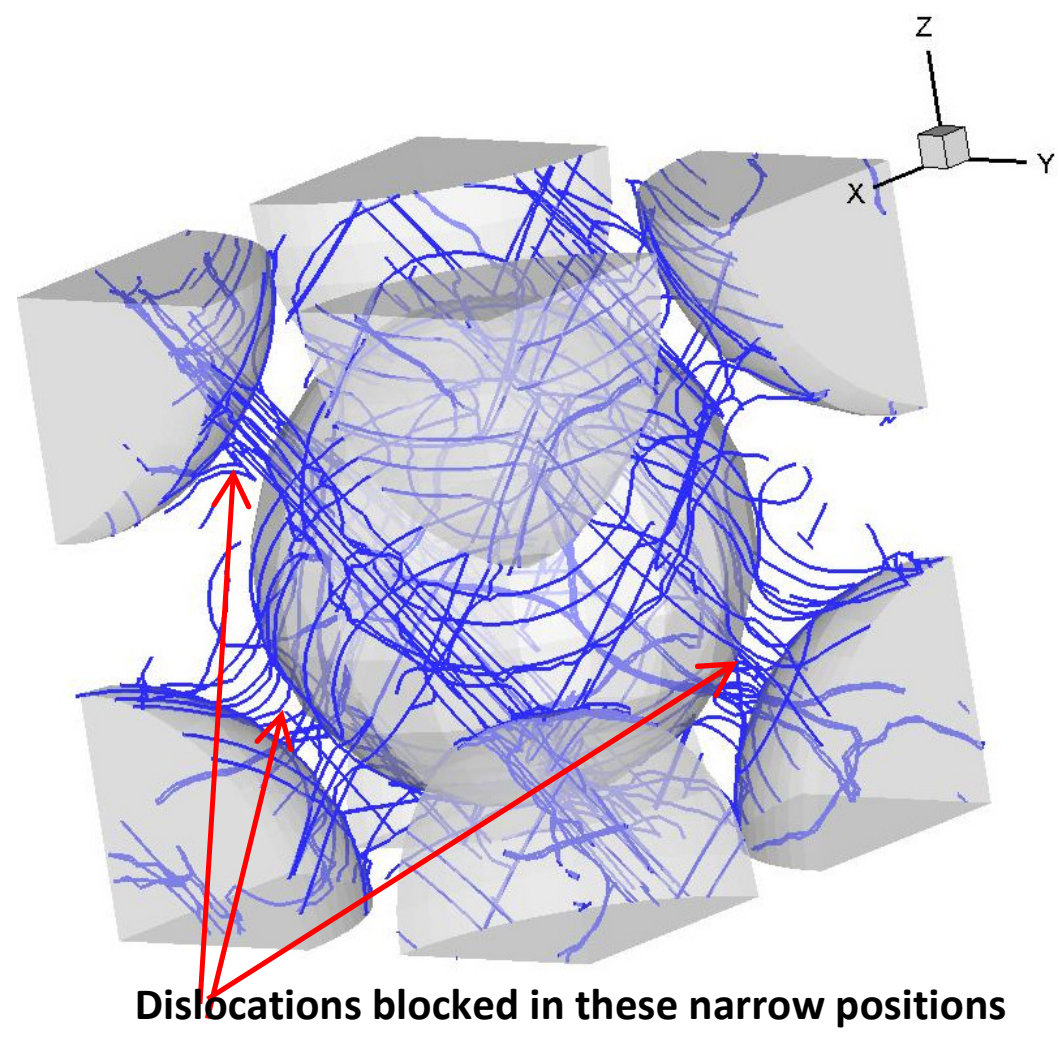

Fig. 13 Dislocation microstructure formed around spherical precipitates, where dislocations are blocked at the narrowest parts of the channel between two neighbouring precipitates. 


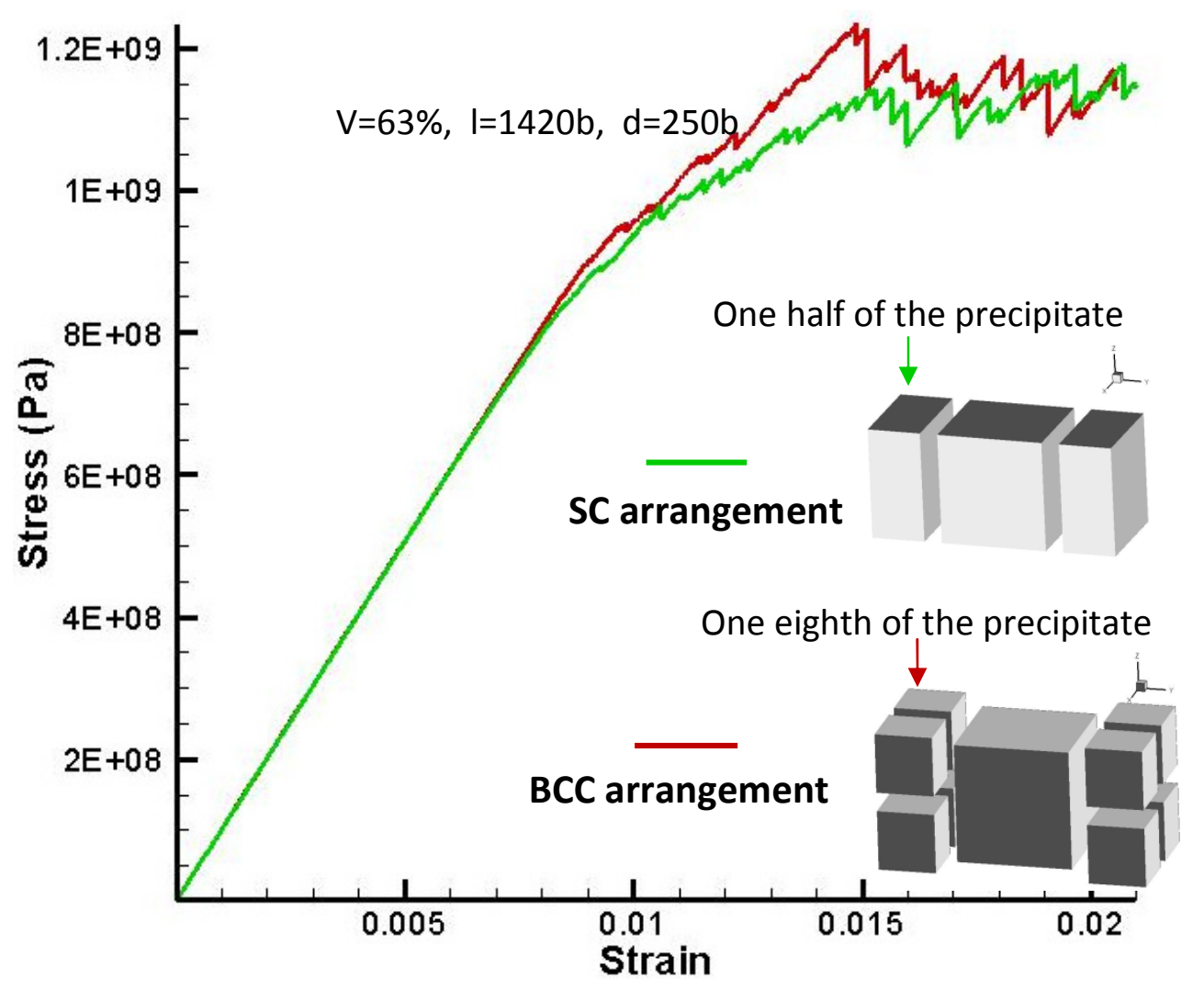

Fig. 14 The influence of precipitate arrangement on the stress-strain response. 


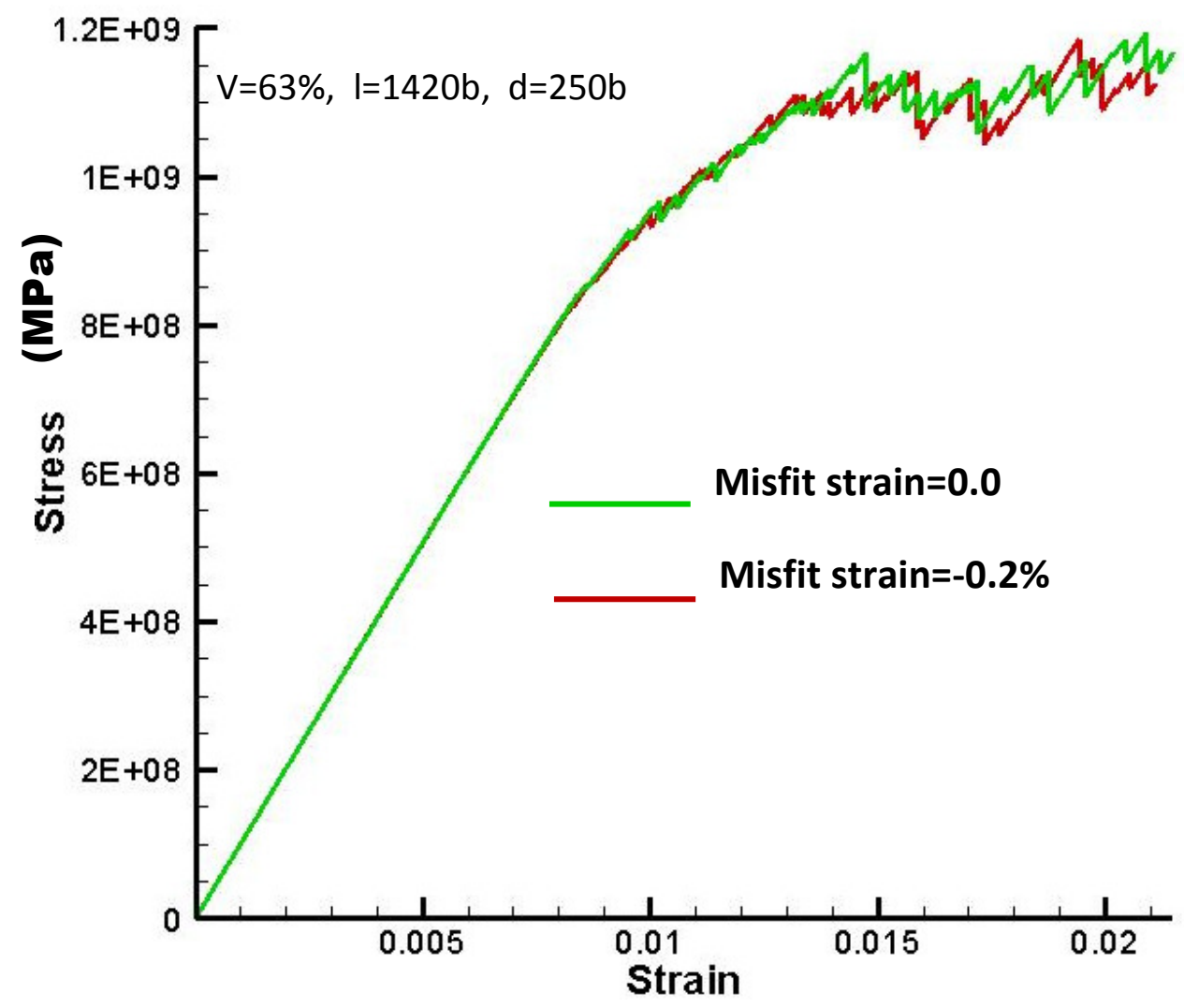

Fig. 15 The stress-strain response with and without lattice misfit strain. 


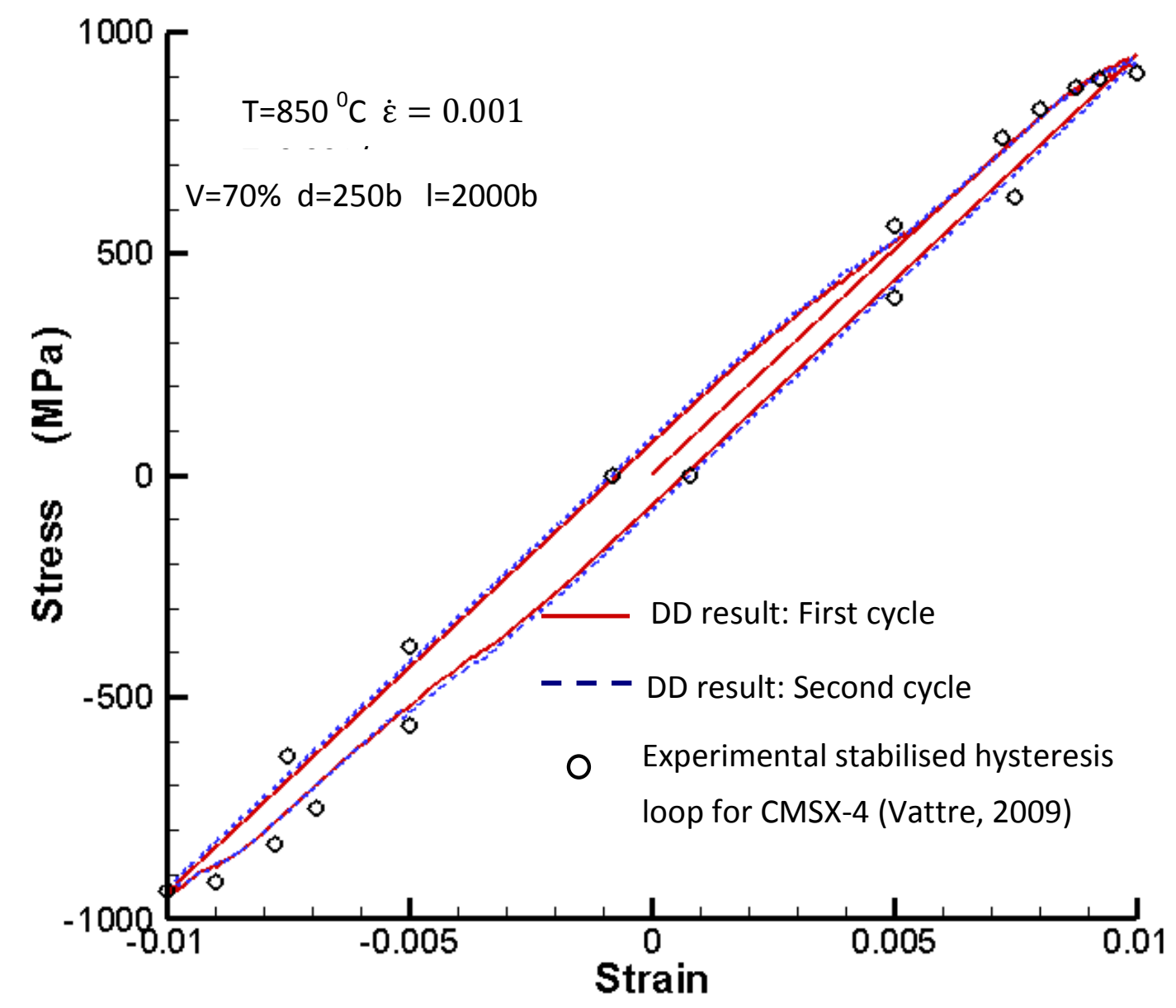

Fig. 16 The simulated stress-strain response for the first two cycles, with comparison against the experimental data for the stabilised hysteresis loop (Vattre, 2009). 